ESAIM: COCV 27 (2021) 68

https://doi.org/10.1051/cocv/2021023
ESAIM: Control, Optimisation and Calculus of Variations

www.esaim-cocv.org

\title{
STOCHASTIC HOMOGENIZATION OF DETERMINISTIC CONTROL PROBLEMS
}

\author{
Alexander VAN-BRUnT*
}

\begin{abstract}
In this paper we study homogenization of a class of control problems in a stationary and ergodic random environment. This problem has been mostly studied in the calculus of variations setting in connection to the homogenization of the Hamilton-Jacobi equation. We extend the result to control problems with more general state dynamics and macroscopically inhomogeneous Lagrangians. Moreover, our approach proves homogenization under weaker growth assumptions on the Lagrangian, even in the well-studied calculus of variations setting.
\end{abstract}

Mathematics Subject Classification. 49J20, 35B27, 49L25.

Received June 21, 2018. Accepted February 24, 2021.

\section{INTRODUCTION}

We study homogenization of deterministic optimal control problems in random ergodic environments with state dynamics beyond the calculus of variations and macroscopically inhomogeneous Lagrangians. We refer the reader to [9] and references therein for general theory on the optimal control problems. The control problem we will be considering is as follows. Let $(\Omega, \mathcal{F}, P)$ be a probability space, $T>0$ be a terminal time and

$$
L:[0, T] \times \mathbb{R}^{d} \times \mathbb{R}^{d} \times \mathbb{R}^{d} \times \Omega \rightarrow \mathbb{R}
$$

be a random field, which we call the Lagrangian. The Lagrangian $L$ will be assumed to be stationary and ergodic with respect to translation in the third argument, see Section 1.4 below for the precise formulation. Fix an $x \in \mathbb{R}^{d}$ and a $t \in(0, T]$. For a given $\omega \in \Omega$, an $\epsilon>0$ and a terminal cost function $\psi(\cdot)$, define the cost functional

$$
J_{\epsilon}(t, x, u, \omega):=\int_{t}^{T} L\left(s, x(s), \frac{x(s)}{\epsilon}, u(s), \omega\right) \mathrm{d} s+\psi(x(T))
$$

over $u \in \mathcal{U}:=L^{\infty}\left([0, T] \rightarrow \mathbb{R}^{d}\right)$, where $x(\cdot)$ is defined by the so-called state dynamics

$$
\left\{\begin{array}{l}
\frac{\mathrm{d} x}{\mathrm{~d} s}(s)=f(x(s), u(s)) \quad \text { for } s \in(t, T], \\
x(t)=x
\end{array}\right.
$$

Keywords and phrases: Optimal control theory, homogenisation, Hamilton Jacobi equations.

Mathematical Institute, University of Oxford, Oxford, OX2 6GG, UK.

* Corresponding author: Alexander.Van-Brunt@maths.ox.ac.uk 
for a suitable $f: \mathbb{R}^{d} \times \mathbb{R}^{d} \rightarrow \mathbb{R}^{d}$. The control problem studies the minimal cost which is called the value function:

$$
V_{\epsilon}(t, x, \omega):=\inf _{u \in \mathcal{U}} J_{\epsilon}(t, x, u, \omega) .
$$

For a fixed $\epsilon>0$, this value function is random depending on $\omega$. Our objective is to prove, under appropriate assumptions, the existence of an effective Lagrangian $\widetilde{L}$ such that

$$
V_{\epsilon}(t, x, \omega) \rightarrow \widetilde{V}(t, x) \text { as } \epsilon \rightarrow 0
$$

almost surely uniformly on compact sets, where $\widetilde{V}$ is the value function corresponding to the cost functional

$$
\widetilde{J}(t, x, u)=\int_{t}^{T} \widetilde{L}(s, x(s), u(s)) \mathrm{d} s+\psi(x(T)),
$$

with the same state dynamics (1.3) as before. This is called homogenization of the control problem.

Under suitable coercivity assumptions, this result automatically implies homogenization of the associated Hamilton-Jacobi equation. Indeed if we define the Hamiltonians

$$
\begin{aligned}
\mathcal{H}\left(t, x, \frac{x(s)}{\epsilon}, p, \omega\right) & =\sup _{v \in \mathbb{R}^{d}}\left\{-f(x, v) \cdot p-L\left(t, x, \frac{x}{\epsilon}, v, \omega\right)\right\}, \\
\widetilde{\mathcal{H}}(t, x, p) & =\sup _{v \in \mathbb{R}^{d}}\{-f(x, v) \cdot p-\widetilde{L}(t, x, v)\}
\end{aligned}
$$

then it is a well known fact that, under our assumptions presented later, the value functions $V_{\epsilon}$ and $\widetilde{V}$ are the unique respective viscosity solutions to the following Hamilton-Jacobi equations:

$$
\begin{aligned}
-\frac{\partial V_{\epsilon}}{\partial t}+\mathcal{H}\left(t, x, \frac{x(s)}{\epsilon}, D_{x} V_{\epsilon}, \omega\right) & =0, \\
-\frac{\partial \widetilde{V}}{\partial t}+\widetilde{\mathcal{H}}\left(t, x, D_{x} \widetilde{V}\right) & =0
\end{aligned}
$$

subject to the terminal conditions $\widetilde{V}(T, x)=V(T, x)=\psi(x)$. Therefore, the convergence (1.5) implies homogenization of the Hamilton-Jacobi equation.

\subsection{Background and earlier results}

Our problem is naturally related to the homogenization of Hamilton-Jacobi equations, which there has been a substantial interest in. The applications of which include large deviations of diffusion process in random environments, front propagation in random media and first passage percolation theory. Such a result has been obtained by several authors. In periodic setting it has been studied via reducing it to a cell problem, as first carried out by [14] for temporally homogeneous Hamiltonians. The result was later extended to the almost periodic case with general Hamiltonians in [10] by using the so called perturbed test function method intiated in [6]. In the stochastic case, these approaches are no longer possible since the corrector does not exist in general [15]. This case was first addressed in $[17,20]$ where the authors applied the subadditive ergodic theorems to the control theoretic interpretation of the solution. It is worth mentioning that in order to find an associated Lagrangian, the Hamiltonian is assumed to be convex. In the non-convex setting, it is known that homogenization does not occur in general $[7,22]$. Later these approaches are further extended to the second order equations with a vanishing viscosity term by $[12,16]$. There is much recent progress in the homogenization of Hamilton-Jacobi 
equations, alot of which has centered around weak KAM theory [4] and metric techniques [1, 3, 5]. For more details, we refer the reader to the introduction in [11].

All the above works mainly focus on the Hamilton-Jacobi equations and the associated control problems are assumed to follow the simplest state dynamics $f(x, u)=u$, a problem called the calculus of variations. The control problem is only in the background and hence there is no reason to consider other complicated state dynamics. However in many real world control problems, it is the Hamilton-Jacobi equation which is in the background and the state dynamics are more general.

For these reasons, we develop a direct approach to prove the homogenization of control problems with general state dynamics. This allows us to obtain the effective Lagrangian and also leads to explicit assumptions in terms of $L$ and $f$. In addition, our approach has two technical merits. First, even in the calculus of variations setting, our assumptions are seen to be less restrictive than the ones in [17,20]. Also, it gives a straightforward way to include macroscopic inhomogeneity to the Lagrangian. Both [17,20] assumed the Hamiltonian to have the form $H\left(x / \epsilon, D_{x} V_{\epsilon}, \omega\right)$. There is a way to extend the results to the macroscopically inhomogeneous cases by using the viscosity solution framework, as carried out in $[14,16]$, but our approach gives an alternative elementary way.

We also hope that the approach of this paper can be extended beyond the scope of the current setting, such as the vanishing viscosity case.

\subsection{Outline of the proof}

In the general setting, we will make a number of assumptions on the Lagrangian $L$ and state dynamics $f$. In order to motivate them, we shall explain a brief outline of the argument.

For simplicity, we shall assume that $\psi(x)=0$ and there exists a Lipschitz continuous optimal control $u^{*}$. The proof of our result is based on a discretization scheme. Specifically to homogenize the control problem, we wish to use a certain ergodic theorem on the object

$$
V_{\epsilon}(t, x, \omega)=\int_{t}^{T} L\left(s, x(s), \frac{x(s)}{\epsilon}, u^{*}(s), \omega\right) \mathrm{d} s
$$

However, this is not straightforward. One immediate reason is that, since we have no a priori information on $u$, the object is not stationary in the "fast variable", $x(\cdot) / \epsilon$, where we expect the averaging to occur. Another issue is that the stationarity and ergodicity of $L$ is assumed only for fixed $(s, x(s))$. We may attempt to cope with this by introducing a discretization. For $\tau>0$ let $t_{0}=t$ and $t_{i}=\left(t_{i-1}+\tau\right) \wedge T$ until the first $i$ such that $t_{i}=T$. This sequence $\left\{t_{i}\right\}_{i=1}^{N}$ defines a partition of $[t, T]$. Define $\bar{x}(\cdot):[t, T) \rightarrow \mathbb{R}^{d}$ as the piecewise linear function with $\bar{x}\left(t_{i}\right)=x\left(t_{i}\right)$ for each $1 \leq i \leq N$ and consider

$$
\sum_{i=1}^{N} \int_{t_{i}}^{t_{i+1}} L\left(t_{i}, x\left(t_{i}\right), \frac{\bar{x}(s)}{\epsilon}, u^{*}\left(t_{i}\right), \omega\right) \mathrm{d} s
$$

Since $\bar{x}(\cdot)$ is a straight line segment on $\left[t_{i}, t_{i+1}\right)$ and the other variables of $L$ are frozen, there is no problem in applying the ergodic theorem on each $\left[t_{i}, t_{i+1}\right)$. If there were no fast variable, it is an easy exercise to show that (1.12) approximates the value function $V_{\epsilon}$ well.

If however we applied this reasoning to our case of interest we would only conclude

$$
V_{\epsilon}(t, x, \omega)=\sum_{i=1}^{N} \int_{t_{i}}^{t_{i+1}} L\left(t_{i}, x\left(t_{i}\right), \frac{\bar{x}(s)}{\epsilon}, u^{*}\left(t_{i}\right), \omega\right) \mathrm{d} s+\mathcal{O}(1) .
$$

Then we see such an approximation would not be useful. The issue is that no matter how small $|x(s)-\bar{x}(s)|$ is, it will be magnified by $\epsilon^{-1}$ and hence we cannot conclude that the values of the Lagrangian are close. This is 
not counterintuitive since we expect the control to fluctuate more wildly as the trajectory attempts to navigate through the random environment.

To address this concern, we instead discretize the control problem in a way which makes no attempt to approximate the controls on the microscopic level, but rather approximates the controls on a macroscopic level and implicitly optimizes over the microscopic environment. This corresponds to introducing the following objects.

1. For some $i<N$ and $u \in \mathcal{U}$, let $\widehat{x}(\cdot)$ follow the frozen dynamics $\frac{\mathrm{d} \widehat{x}(s)}{\mathrm{d} s}=f\left(x\left(t_{i}\right), u(s)\right)$ for $s \in\left[t_{i}, t_{i+1}\right)$ with $\widehat{x}\left(t_{i}\right)=x\left(t_{i}\right)$.

2. Suppose there exists $\widetilde{u}_{i}$ such that

$$
f\left(x\left(t_{i}\right), \widetilde{u}_{i}\right)=\frac{x\left(t_{i+1}\right)-x\left(t_{i}\right)}{\tau}
$$

then define the set

$$
\Pi_{t_{i}, x\left(t_{i}\right), \tau, \widetilde{u}_{i}}=\left\{u \in \mathcal{U}: \int_{t_{i}}^{t_{i+1}} f\left(x\left(t_{i}\right), u(s)\right) \mathrm{d} s=\tau f\left(x\left(t_{i}\right), \widetilde{u}_{i}\right)\right\} .
$$

3. Let

$$
L_{\tau, \epsilon}\left(t_{i}, x\left(t_{i}\right), \widetilde{u}_{i}, \omega\right):=\inf _{u \in \Pi_{t_{i}, x\left(t_{i}\right), \tau, \tilde{u}_{i}}} \int_{t_{i}}^{t_{i+1}} L\left(t_{i}, x\left(t_{i}\right), \frac{x(s)}{\epsilon}, u(s), \omega\right) \mathrm{d} s .
$$

This definition requires some explanation. The $\widetilde{u}_{i}$ is superfluous since by substituting its defining equation to (1.15), we see that $L_{\tau, \epsilon}$ is simply the 'cheapest cost' to get from $x\left(t_{i}\right)$ to $x\left(t_{i+1}\right)$. In the special case of the calculus of variations, this $\widetilde{u}_{i}$ is given simply as $\tau^{-1} \int_{t_{i}}^{t_{i+1}} u^{*}(s) \mathrm{d} s$, i.e., the average direction of the trajectory over the time $\tau$. In the general case, we will need a convexity assumption on the image of $f\left(x\left(t_{i}\right), \cdot\right)$ to guartanee the existence of such an $\widetilde{u}_{i}$.

Now if we could show that the use of frozen dynamics has little effect to the cost, i.e.,

$$
L_{\tau, \epsilon}\left(t_{i}, x\left(t_{i}\right), \widetilde{u}_{i}, \omega\right) \sim \int_{t_{i}}^{t_{i+1}} L\left(s, x^{*}(s), \frac{x^{*}(s)}{\epsilon}, u^{*}(s)\right) \mathrm{d} s
$$

then we obtain the following improvement of (1.13):

$$
V_{\epsilon}(t, x, \omega)=\sum_{i=1}^{N} L_{\tau, \epsilon}\left(t_{i}, x\left(t_{i}\right), \widetilde{u}_{i}, \omega\right)+o(1) .
$$

Thanks to the frozen dynamics, we can show that $L_{\tau, \epsilon}$ is a stationary subadditive process. This allows us to use the subadditive ergodic theorem to deduce the almost sure existence of the limit

$$
\lim _{\epsilon \rightarrow 0} L_{\tau, \epsilon}\left(t_{i}, x\left(t_{i}\right), \widetilde{u}_{i}, \omega\right)=\widetilde{L}_{\tau}\left(t_{i}, x\left(t_{i}\right), \widetilde{u}\right)
$$

which is positively homogeneous in $\tau$. We can then define an effective Lagrangian by

$$
\widetilde{L}\left(t_{i}, x\left(t_{i}\right), \widetilde{u}_{i}\right)=\frac{\widetilde{L}_{\tau}\left(t_{i}, x\left(t_{i}\right), \widetilde{u}_{i}\right)}{\tau} .
$$


If we know that $\widetilde{L}$ is jointly continuous, combined with the assumed "regularity" of $u^{*}$, we can proceed as

$$
V_{\epsilon}(t, x, \omega) \sim \sum_{i=1}^{N}\left(t_{i+1}-t_{i}\right) \widetilde{L}\left(t_{i}, x\left(t_{i}\right), u^{*}\left(t_{i}\right)\right) \sim \int_{t}^{T} \widetilde{L}\left(s, x(s), u^{*}(s)\right) \mathrm{d} s \geq \widetilde{V}(t, x)
$$

The proof of the other direction is done by reserving the above procedure.

Let us comment on several key points in the above outline. First of all, the use of subadditive ergodic theorem is not new in this context. It is used in $[17,20]$ and many later works also rely on it. So the additional difficulties in the generality that we present here is the regularity issues: the existence of "regular" approximate optimal control, the stability of $\widetilde{L}$ in modification (freezing) of the state dynamics, and the continuity of $\widetilde{L}$. For the first issue, we will simply show that there is a 'nearly step function' control that approximates our value function. The second and third issues are more subtle and closely related. The difficulty is highlighted by the fact that we will want to prove that the effective Lagrangian $\widetilde{L}$ is continuous via proving a equicontinuity result on $\widetilde{L}_{\tau, \epsilon}$. As we have seen in (1.13), the cost functional

$$
\int L\left(s, x(s), \frac{x(s)}{\epsilon}, u(s), \omega\right) \mathrm{d} s
$$

is highly unstable in $x(\cdot)$. Thus in order to compare the two costs with different starting points, we cannot use a simple continuity argument. Instead we construct controls for which one trajectory essentially traces out the other one exactly and hence acquires no error in the microscopic variable. Certainly, this type of argument requires some flexibility to the state dynamics. In addition to this, since we will assume the continuity of $L$ only locally uniformly in the control variable, it is also important to introduce a type of effective compactness of the control space which ensures that the control does not get too large. This requires a coercivity assumption.

\subsection{Assumptions and main results}

We first recall the definition of stationarity and ergodicity. Let $\left\{\varphi_{x}: \Omega \rightarrow \Omega\right\}_{x \in \mathbb{R}^{d}}$ be a group of measure preserving transformations on the probability space $(\Omega, \mathcal{F}, P)$, that is for any $x, y \in \mathbb{R}^{d}, \varphi_{x+y}=\varphi_{x} \circ \varphi_{y}$ and $P \circ \varphi_{x}^{-1}=P$. The transformation group $\varphi$ is said to be ergodic if $P(A) \in\{0,1\}$ for all $A \in \mathcal{F}$ such that $\varphi_{y}^{-1} A=A$ up to a null-set for all $y \in \mathbb{R}^{d}$.

Assumption 1.1. For every fixed $t_{0} \in[0, T)$ and $\left(x_{0}, u\right) \in \mathbb{R}^{d} \times \mathbb{R}^{d}$ the process $L\left(t_{0}, x_{0}, \cdot, u, \omega\right)$ is stationary ergodic in $\Omega$. That is to say there is an ergodic transformation group $\left\{\varphi_{x}: \Omega \rightarrow \Omega\right\}_{x \in \mathbb{R}^{d}}$ such that, for every $x, y$

$$
L\left(t_{0}, x_{0}, x+y, u, \omega\right)=L\left(t_{0}, x_{0}, x, u, \varphi_{y} \omega\right)
$$

For the remaining assumptions, we make the following definitions

$$
\begin{aligned}
U^{R}: & =\left\{u \in \mathbb{R}^{d}:|u| \leq R\right\}, \\
\mathcal{U}^{R} & =\left\{u \in L^{\infty}\left([0, T] \rightarrow \mathbb{R}^{d}\right), \quad\|u\|_{\infty} \leq R\right\} .
\end{aligned}
$$

and use this to define the estimates on the cost function and state dynamics

$$
\begin{aligned}
L^{*}(R) & :=\sup _{t, x, y, u \in U^{R}, \omega \in \Omega}|L(t, x, y, u, \omega)|, \\
L_{\mathrm{inf}}(R) & :=\inf _{t, x, y,|u| \geq R, \omega \in \Omega} L(t, x, y, u, \omega), \\
f^{*}(R) & :=\sup _{x, u \in U^{R}}|f(x, u)| .
\end{aligned}
$$


Assumption 1.2. For every $R>0$, the terms $L^{*}(R), L_{\text {inf }}(R)$ and $f^{*}(R)$ are finite.

Assumption 1.3. $f$ is uniformly Lipschitz continuous on each $\mathbb{R}^{d} \times U^{R}$ with Lipschitz constant $\|f\|_{\text {Lip }}^{R}$.

Assumption 1.4. For every $R>0$, there exists a constant $\|L\|_{L i p}^{R}$ and a continuous function $m_{L}^{R}:[0, \infty) \rightarrow$ $[0, \infty)$ with $m_{L}^{R}(0)=0$ such that for every $\left(x_{i}, y_{i}\right) \in \mathbb{R}^{d} \times \mathbb{R}^{d}$ and $u_{i} \in U^{R}, t_{i} \in[0, T]$, for $i \in\{1,2\}$,

$$
\left|L\left(t, x_{1}, y_{1}, u_{1}, \omega\right)-L\left(t, x_{2}, y_{2}, u_{2}, \omega\right)\right| \leq m_{L}^{R}\left(\left|x_{1}-x_{2}\right|+\left|y_{1}-y_{2}\right|\right)+\|L\|_{L i p}^{R}\left(\left|u_{1}-u_{2}\right|\right)
$$

almost surely in $\Omega$. Furthermore we assume that there exists a $\|L\|_{L i p}$ such that

$$
\left|L\left(t_{1}, x, y, u, \omega\right)-L\left(t_{2}, x, y, u, \omega\right)\right| \leq\|L\|_{L i p}\left(\left|t_{1}-t_{2}\right|\right)
$$

for all $t, s \in[0, T]$ and $(x, y, u) \in \mathbb{R}^{d} \times \mathbb{R}^{d} \times \mathbb{R}^{d}$ a.s. in $\Omega$.

Assumption 1.5. $\psi$ is bounded and there exists a $m^{\psi}:[0, \infty) \rightarrow[0, \infty)$ with $m_{\psi}(0)=0$ such that

$$
|\psi(x)-\psi(y)| \leq m^{\psi}(|x-y|)
$$

Assumption 1.6. For some $\widetilde{M}>0$, for every $x \in \mathbb{R}^{d}$ there exists a $u \in U^{\widetilde{M}}$ such that

$$
f(x, u)=0 .
$$

Assumption 1.7. There exists an $L_{*}(u) \leq L_{\text {inf }}(|u|)$ and $\lambda>0$ such that

$$
\frac{L_{*}(u)}{f^{*}(|u|+\lambda)}=: \gamma(u) \rightarrow \infty \text { as }|u| \rightarrow \infty
$$

Furthermore there exists a function $\Theta: \mathbb{R}^{d} \rightarrow \mathbb{R}^{d}$ such that, for every $\left(x, u_{1}, u_{2}\right) \in \mathbb{R}^{d} \times \mathbb{R}^{d} \times \mathbb{R}^{d}$

$$
L_{*}\left(u_{1}\right)-L_{*}\left(u_{2}\right) \geq \Theta\left(u_{2}\right) \cdot\left[f\left(x, u_{1}\right)-f\left(x, u_{2}\right)\right]
$$

For $\eta>0$ we denote the open ball of radius $\eta$ around $x$ as $B_{\eta}(x)$.

Assumption 1.8. For any given $R>0$ and every $x_{0} \in \mathbb{R}^{d}$ and $u_{0} \in U^{R}$, there exists an $\eta(R)>0$ independent of $x_{0}$ and a Lipschitz continuous function

$$
H: B_{\eta(R)}\left(x_{0}\right) \times B_{\eta(R)}\left(f\left(x_{0}, u_{0}\right)\right): \rightarrow B_{\eta(R)}\left(u_{0}\right)
$$

such that $H\left(x_{0}, f\left(x_{0}, u_{0}\right)\right)=u_{0}$ and for any $(x, v) \in B_{\eta(R)}\left(x_{0}\right) \times B_{\eta(R)}\left(f\left(x_{0}, u_{0}\right)\right)$

$$
f(x, H(x, v))=v .
$$

This function $H$ depends on $x_{0}$ and $u_{0}$, which we omit in our notation. We assume this family of functions $H$ parametrised by elements of $\mathbb{R}^{d} \times U^{R}$ is Lipschitz equicontinuous, and denote this modulus of continunity by $\|H\|_{\text {Lip }}^{R}$.

Assumption 1.9. For each $R>0$ and every $x \in \mathbb{R}^{d}, f\left(x, U^{R}\right)$ is a convex set.

Our main results concerning homogenization of control problems and Hamilton Jacobi equations are as follows. 
Theorem 1.10. Under Assumptions 1.1-1.9 there exists an effective Lagrangian $\widetilde{L}$ such that

$$
\lim _{\epsilon \rightarrow 0} V_{\epsilon}(t, x, \omega)=\widetilde{V}(t, x)
$$

almost surely in $\Omega$, uniformly on compact sets, where $\widetilde{V}$ is the value function corresponding to the cost functional

$$
\widetilde{J}(t, x, u)=\int_{t}^{T} \widetilde{L}(s, x(s), u(s)) \mathrm{d} s+\psi(x(T))
$$

and state dynamics

$$
\frac{\mathrm{d} x(s)}{\mathrm{d} s}=f(x(s), u(s))
$$

Theorem 1.11. Under Assumptions 1.1-1.9 there exists an effective Hamiltonian $\widetilde{H}$ such that

$$
\lim _{\epsilon \rightarrow 0} V_{\epsilon}(t, x, \omega)=\widetilde{V}(t, x)
$$

almost surely in $\Omega$. uniformly on compact sets, where $\widetilde{V}(t, x)$ is the unique viscosity solution to the Hamilton Jacobi equation

$$
-\frac{\partial \widetilde{V}(t, x)}{\partial t}+\widetilde{H}\left(t, x, D_{x} \widetilde{V}\right)=0, \quad V(T, x)=\psi(x) .
$$

Assumption 1.1 is a standard stationary ergodic assumption. Assumption 1.2 ensures that the control problem is well-posed for each $\epsilon>0$. Similarly Assumption 1.3 is a standard assumption to ensure the solution to the integral equation

$$
x(t)-x\left(t_{0}\right)=\int_{t_{0}}^{t} f(x(s), u(s)) \mathrm{d} s
$$

is well defined for each $u \in \mathcal{U}$.

The continuity conditions in Assumption 1.4 and are used to prove effective boundedness as per Lemmas 2.2 and 2.3. In fact this is the only place that Lipschitz continunity is needed and the proof of Lemmas 2.2 and 2.3 extensively rely on it.

The combination of Assumption 1.6 and 1.8 imply that there exists a $\delta>0$ such that at each $x$, for every $v \in \mathbb{S}^{d}$ (the d-dimensional unit sphere) there is a $u \in U^{M}$ such that

$$
f(x, u)=\delta v
$$

for $M:=\widetilde{M}+\eta(\widetilde{M})$. This is one form of the 'flexibility' mentioned in Section 1.2 which is important to stipulate. The terms $M$ and $\delta$ will be referred to throughout.

Assumption 1.7 is analogous to various coercive assumptions in the calculus of variations setting. The implication of Assumption 1.7 is that we can essentially compactify the control set. This is a key point in the analysis. The presence of the $\lambda>0$ makes this a mildly stronger assumption, however is satisfied in "many cases". For example, in the calculus of variations with $L_{*}(u) \geq c|u|^{\beta}-C$ for $\beta>1$ and appropriate constants $c$ and $C$. The role of the second statement in this assumption is to prove that the homogenized Lagrangian satisfies the same coercive assumption as the original Lagrangian. It is essentially a generalization of convexity. In fact in 
the calculus of variations if we assume that $L_{*}(u)$ is a differentiable convex function, then the second statement of Assumption 1.7 is satisfied by setting $\Theta(u):=\nabla L_{*}(u)$. This is discussed further in Section 2.5.

Assumptions 1.6 and 1.8 give us flexibility but it in fact implies more, e.g.. given any control, we can always find another control which follows the same trajectory but at a higher speed. Its formulation is motivated by implicit function type theorems. A natural setting where Assumption 1.8 holds is when $f$ is continuously differentiable in the control variable with an Jacobian whose inverse is uniformly bounded on $\mathbb{R}^{d} \times U^{R}$ for each $R>0$. The function $H$ appearing in Assumption 1.8 will also be referenced throughout, with the dependence on $x_{0}, u$ omitted - they will always be clear from the context. Similarly to Assumption 1.3, the Lipschitz continuity of $H$ is necessary for the proof of Lemmas 2.2 and 2.3.

The assumptions we impose on the state dynamics are strong however is more general then the calculus of variations. Assumption 1.8 and 1.9 will imply the existence of the $\widetilde{u}$ as in equation (1.14), which is essential to preserve the state dynamics. Although restrictive, Assumption 1.9, is a common assumption in the theory of optimal control. A typical result is that under Assumption 1.9, suitable regularity conditions, and compactness (or effective compactness as per Lem. 2.2) of the control set, an optimal control exists. We refer the reader to $[8,18,21]$ for more details, though we emphasise that the existence of an optimal control does not play a role in the argument presented here.

Remark 1.12. Let us compare our assumptions to those in $[17,20]$ in their setting. Thus we focus on the case of calculus of variations, which makes Assumptions 1.3, 1.6 and 1.8 trivial. Assume that $L$ is independent of the first and second arguments. Then Assumption 1.7 is equivalent to a mean coercivity condition which states that $L_{*}(u)$ can be taken as a convex function satisfying $\lim _{|u| \rightarrow \infty} L_{*}(u) /|u|=\infty$, which both $[17,20]$ assume. But in fact additional polynomial growth conditions are assumed in [20] (upper and lower bounds) and [17] (upper bound), which we do not require. Concerning Assumptions 1.4, note first that in this case, we may assume that $L$ is convex in $u$ since its convex biconjugate leads to the same Hamiltonian and hence same value function. Combined with the above polynomial upper bounds, the local Lipschitz continuity in $u$ follows. Hence it is also implicitly assumed in $[17,20]$. Therefore our regularity assumption is no stronger than theirs, and [17] moreover assumes continuous differentiability of $L$ in $u$.

\subsection{Examples}

The simplest example is the calculus of variations setting with the Lagrangian given by

$$
L\left(\frac{x}{\epsilon}, v, \omega\right)=\frac{1}{2}|v|^{2}-\Phi\left(\frac{x(s)}{\epsilon}, \omega\right)
$$

for some $\Phi(\cdot, \cdot): \mathbb{R}^{d} \times \Omega \rightarrow \mathbb{R}$. We assume that $\Phi$ is almost surely uniformly continuous and bounded. This type of Lagrangian is referred to as an action in a random potential. Our result readily applies to this. However we will consider a far more involved example motivated by the theory of relativity, which fully displays the scope of our work. A principle complication in this setting is that the velocity, $v$, (with respect to coordinate time), is bounded by the so call 'light cones', which may depend on position. Thus implicitly we are no longer in the true calculus of variations setting, but restricted to a space-time manifold.

For definiteness we will consider the Lagrangian given by

$$
L\left(\frac{x}{\epsilon}, v, \omega\right)=-m c^{2}\left((1-\alpha(x))-\frac{v^{2}}{c^{2}}(1-\alpha(x))^{-1}\right)^{1 / 2}+\frac{\gamma v^{2}}{(1-\alpha(x))^{2}-\frac{|v|^{2}}{c^{2}}}-\Phi\left(\frac{x}{\epsilon}, \omega\right),
$$

where $\gamma>0$ and

$$
\alpha(x)=\frac{1}{\sqrt{|x|^{2}+w^{2}}} .
$$


for a given $w>1$. This is motivated by the Schwarzchild geometry in general relativity. We have significantly simplified it for the sake of exposition. The state dynamics is as in the calculus of variations, i.e taken as $f(x, v)=v$.

Note that the Lagrangian becomes undefined outside of the 'light cone' regions which depend on $x$, specifically when

$$
v \geq c(1-\alpha(x))
$$

This means that the direct approach of the calculus of variations is difficult. However in our framework, this can be done with ease. Let us define

$$
u:=H(x, v)=\frac{v}{\sqrt{(1-\alpha(x))^{2}-\frac{|v|^{2}}{c^{2}}}} .
$$

This can be inverted for $v$

$$
v=\frac{u(1-\alpha(x))}{\sqrt{1+\frac{|u|^{2}}{c^{2}}}} .
$$

We can then rewrite the Lagrangian in terms of $v$ to get

$$
L\left(\frac{x}{\epsilon}, u, \omega\right)=-m c^{2}\left(\frac{(1-\alpha(x))}{1+\frac{|u|^{2}}{c^{2}}}\right)^{1 / 2}+\gamma|u|^{2}-\Phi\left(\frac{x}{\epsilon}, \omega\right)
$$

Then we can define the control problem associated to the cost function

$$
J_{\epsilon}(t, x, u, \omega)=-m c^{2} \int_{t}^{T}\left[\left(\frac{(1-\alpha(x))}{1+\frac{|u(s)|^{2}}{c^{2}}}\right)^{1 / 2}+\gamma|u(s)|^{2}-\Phi\left(\frac{x(s)}{\epsilon}, \omega\right)\right] \mathrm{d} s
$$

with state dynamics

$$
\frac{\mathrm{d} x(s)}{\mathrm{d} s}=\frac{u(s)(1-\alpha(x))}{\sqrt{1+\frac{|u(s)|^{2}}{c^{2}}}}=: f(x(s), u(s)) .
$$

With this change of variables, we have a control problem in which the control argument is well defined on all of $\mathbb{R}^{d}$ and satisfies all of our assumptions. In particular, we note that the image of the state dynamics function $f$ is bounded by the 'light cones'. In addition, at each $x \in \mathbb{R}^{d}$, the state dynamics are isotropic in the control variable which ensures that Assumption 1.9 is satisfied. Indeed isotropy is one common setting in which Assumption 1.9 can be expected to hold.

We apply our homogenization result to conclude that there exists an effective Lagrangian $\widetilde{L}$ such that

$$
\lim _{\epsilon \rightarrow 0} V_{\epsilon}(t, x, \omega)=\widetilde{V}(t, x),
$$

where

$$
\widetilde{V}(t, x)=\inf _{u \in \mathcal{U}} \int_{t}^{T} \widetilde{L}(x(s), u(s)) \mathrm{d} s,
$$


and $x(s)$ satisfies the state dynamics

$$
\frac{\mathrm{d} x(s)}{\mathrm{d} s}=f(x(s), u(s))
$$

We see then that the power of our homogenization result is that it can be applied to situations in which the state dynamics non-trivially depend on the location. With further analysis, one can consider more complicated Lorentzian geometries.

Remark 1.13. We emphasise that, because our homogenization result has preserved the state dynamics, we can now readily convert this back into a calculus of variations problem by using (1.48).

Remark 1.14. In this example, we have an explicit formula for the function $H(x, v)$ appearing in Assumption 1.8;

$$
H(x, v)=\frac{v}{\sqrt{(1-\alpha(x))^{2}-\frac{|v|^{2}}{c^{2}}}} .
$$

in fact, precisely by (1.48)-(1.49), we have that $f(x, H(x, v))=v$ and $H(x, f(x, u))=u$.

\section{Proof of main Results}

\subsection{Approximation scheme}

We define the approximation scheme which is the key construction in the proof of main result. This is based on defining a discrete cost function by locally optimizing over a given terminal condition. Fix a $\left(t_{0}, x_{0}\right) \in \mathbb{R}^{d} \times \mathbb{R}^{d}$ and a time step $\tau>0$. For $i \geq 1$ let $t_{i}=\left(t_{i-1}+\tau\right) \wedge T$ until the first $N$ such that $t_{N}=T$. This sequence $\left\{t_{i}\right\}_{i=1}^{N}$ defines a partition of $\left[t_{0}, T\right]$. For a given $\widetilde{u}_{0} \in \mathbb{R}^{d}$ we recall the definition

$$
L_{\tau, \epsilon}\left(t_{0}, x_{0}, \widetilde{u}_{0}, \omega\right):=\inf _{u(s) \in \Pi_{t_{0}, x_{0}, \tau, \tilde{u}_{0}}} \int_{t_{0}}^{t_{0}+\tau} L\left(t_{0}, x_{0}, \frac{x(s)}{\epsilon}, u(s), \omega\right) \mathrm{d} s
$$

where

$$
\Pi_{t_{0}, x_{0}, \tau, \widetilde{u}_{0}}=\left\{u \in \mathcal{U}: \int_{t_{0}}^{t_{0}+\tau} f\left(x_{0}, u(s)\right) \mathrm{d} s=\tau f\left(x_{0}, \widetilde{u}_{0}\right)\right\}
$$

The set $\Pi_{t_{0}, x_{0}, \tau, \widetilde{u}_{0}}$ will never be empty. In particular the constant control given by $u(s)=\widetilde{u_{0}}$ is in $\Pi_{t_{0}, x_{0}, \tau, \widetilde{u}_{0}}$. Hence $L_{\tau, \epsilon}$ is always well defined. Note that in these objects, all the macroscopic variables are frozen. This is to ensure stationarity in preparation of utilizing the sub-additive ergodic theorem.

For this fixed $\left(t_{0}, x_{0}\right)$, if we take a finite sequence of vectors $\left\{\widetilde{u}_{i}\right\}_{i=0}^{N-1}$, where each $\widetilde{u}_{i} \in \mathbb{R}^{d}$, we can define the discrete trajectory sequence $\left\{x_{i}\right\}_{i=0}^{N}$ via the discrete state dynamics

$$
x_{i+1}=x_{i}+\tau f\left(x_{i}, \widetilde{u}_{i}\right) .
$$

Let $\mathcal{U}_{\tau}$ denote the set of all such sequences $\left\{\widetilde{u}_{i}\right\}_{i=0}^{N-1}$. This $\mathcal{U}_{\tau}$ is our discrete approximation to $\mathcal{U}$. For $\widetilde{u}=\left\{\widetilde{u}_{i}\right\}_{i=0}^{N-1} \in \mathcal{U}_{\tau}$ define an approximate effective cost functional

$$
J_{\tau, \epsilon}\left(t_{0}, x_{0}, \widetilde{u}, \omega\right)=\sum_{i=0}^{N-1} L_{\tau, \epsilon}\left(t_{i}, x_{i}, \widetilde{u}_{i}, \omega\right)+\psi\left(x_{N}\right)
$$


and approximate effective value function

$$
V_{\tau, \epsilon}\left(t_{0}, x_{0}, \omega\right)=\inf _{\widetilde{u} \in \mathcal{U}_{\tau}} J_{\tau, \epsilon}\left(t_{0}, x_{0}, \widetilde{u}, \omega\right)
$$

We also define the non-stationary version of $L_{\tau, \epsilon}$ as

$$
\widehat{L}_{\tau, \epsilon}\left(t_{0}, x_{0}, \tau, \widetilde{u}_{0}, \omega\right):=\inf _{u(s) \in \widehat{\Pi}_{t_{0}, x_{0}, \tau, \tilde{u}_{0}}} \int_{t_{0}}^{t_{0}+\tau} L\left(s, x(s), \frac{x(s)}{\epsilon}, u(s), \omega\right) \mathrm{d} s,
$$

where

$$
\widehat{\Pi}_{t_{0}, x_{0}, \tau, \widetilde{u}_{0}}=\left\{u \in \mathcal{U}: \int_{t_{0}}^{t_{0}+\tau} f(x(s), u(s)) \mathrm{d} s=\tau f\left(x_{0}, \widetilde{u}_{0}\right)\right\}
$$

Note that, given a $\widetilde{u}_{0}$, if we take $\tau$ sufficiently small such that $\tau \mid f^{*}\left(\left|\widetilde{u}_{0}\right|\right) \leq \eta\left(\left|\widetilde{u}_{0}\right|\right)$ then, by Assumption 1.8, there will exist a $\widehat{u}_{0}(\cdot) \in \widehat{\Pi}_{t_{0}, x_{0}, \tau, \widetilde{u}_{0}}$ such that, for all $s \in\left[t_{0}, t_{0}+\tau\right)$,

$$
f\left(x(s), \widehat{u}_{0}(s)\right)=f\left(x_{0}, \widetilde{u}_{0}\right) .
$$

In particular, given a $K>0$ and a $\widetilde{u}_{0} \in \mathcal{U}^{K}$, we can take $\tau$ so small (depending only on $K$ ), such that the set $\widehat{\Pi}_{t_{0}, x_{0}, \tau, \widetilde{u}_{0}}$ is non empty and hence in this regime, $\widehat{L}_{\tau, \epsilon}$ is always well defined.

\subsection{Technical lemmas}

Throughout the proof of the main theorem, we will require several technical lemmas. The proof of these is relegated to Section 3. The first of these is regarding the use of approximating controls with piecewise constant dynamics.

We consider a general control problem $\bar{f}(x, u)$ and Lagrangian $\bar{L}(t, x, u)$. For this control problem we define $\mathcal{S}$ be the set of controls such that $\bar{f}(x(s), u(s))$ is a step function. That is, $\mathcal{S}$ is the set of controls such that for some intervals $\left\{\left[t_{i}, t_{i+1}\right)\right\}_{i=1}^{N}$ partitioning $\left[t_{0}, \bar{T}\right]$ and values $\left\{v_{i}\right\}_{i=1}^{N}$,

$$
\bar{f}(x(r), u(r))=\sum_{i=0}^{N} v_{i} 1_{\left[t_{i}, t_{i+1}\right)}(r) .
$$

We will always assume that $\bar{f}, \bar{L}$ satisfies Assumptions 1.1-1.9, with the bounds $f^{*}, L^{*}$. The following lemma will be applied to multiple control problems.

Lemma 2.1. Let $\bar{J}$ and $\bar{V}$ be the cost and value function of a control problem on time $[0, \bar{T}]$ with Lagrangian $\bar{L}$ and state dynamics $\bar{f}$. Assume that $\bar{L}$ and $\bar{f}$ satisfy Assumptions 1.1-1.9. Then for every $t \in[0, \bar{T}]$ and $x \in \mathbb{R}^{d}$

$$
\bar{V}(t, x):=\inf _{u \in \mathcal{U}} \bar{J}(t, x, u)=\inf _{u \in \mathcal{S}} \bar{J}(t, x, u) .
$$

The same is true for the control problem with terminal constraint $x(\bar{T})=x$.

The second lemma argues that we can, without loss of generality, restrict the control space to a compact set. To formalize this we consider the cost functions obtained by restricting the admissible controls to $\mathcal{U}^{R}$ for a fixed $R>0$ and hence define for each $(t, x, \omega) \in[0, T], \times \mathbb{R}^{d} \times \Omega$

$$
V_{\epsilon}^{R}(t, x, \omega):=\inf _{u \in \mathcal{U}^{\mathcal{R}}} J_{\epsilon}(t, x, u, \omega)
$$


and the objects, given $\left(t_{0}, x_{0}, \widetilde{u}_{0}\right) \in[0, T] \times \mathbb{R}^{d} \times \mathbb{R}^{d}$,

$$
L_{\tau, \epsilon}^{R}\left(t_{0}, x_{0}, \widetilde{u}_{0}, \omega\right):=\inf _{u(s) \in \Pi_{t_{0}, x_{0}, \tau, \tilde{u}_{0}} \cap \mathcal{U}^{R}} \int_{t_{0}}^{t_{0}+\tau} L\left(t_{0}, x_{0}, \frac{x(s)}{\epsilon}, u(s), \omega\right) \mathrm{d} s
$$

and

$$
\widehat{L}_{\tau, \epsilon}^{R}\left(t_{0}, x_{0}, \widetilde{u}_{0}, \omega\right):=\inf _{u(s) \in \widehat{\Pi}_{t_{0}, x_{0}, \tau, \tilde{u}_{0}} \cap \mathcal{U}^{R}} \int_{t_{0}}^{t_{0}+\tau} L\left(t_{0}, x_{0}, \frac{x(s)}{\epsilon}, u(s), \omega\right) \mathrm{d} s .
$$

Similarly, for the discrete control problem we define

$$
\mathcal{U}_{\tau}^{R}=\left\{\widetilde{u} \in \mathcal{U}_{\tau}:\left|\widetilde{u}_{i}\right| \leq R \text { for each } 1 \leq i \leq N-1,\right\}
$$

and the value function

$$
V_{\tau}^{R}(t, x, \omega)=\inf _{\widetilde{u} \in \mathcal{U}_{\tau}^{R}} \widetilde{J}_{\tau, \epsilon}(t, x, u, \omega)
$$

Lemma 2.2. There exists an $K>0$ such that for all $t \in[0, T]$ and $x \in \mathbb{R}^{d}, \epsilon>0$ and almost every $\omega$,

$$
V_{\epsilon}^{K}(t, x, \omega)=V_{\epsilon}(t, x, \omega)
$$

and

$$
V_{\tau, \epsilon}^{K}(t, x, \omega)=V_{\tau, \epsilon}(t, x, \omega)
$$

Lemma 2.3. For $\tau>0$ and $\widetilde{u} \in \mathbb{R}^{d}$ such that $\tau f\left(x_{0}, \widetilde{u}\right) \leq \eta(|\widetilde{u}|)$, there exists a $K=K(\widetilde{u})$ such that for all $t_{0} \in[0, T]$ and $x_{0} \in \mathbb{R}^{d}, \epsilon>0$ and almost every $\omega$,

$$
L_{\tau, \epsilon}\left(t_{0}, x_{0}, \widetilde{u}, \omega\right)=L_{\tau, \epsilon}^{K}\left(t_{0}, x_{0}, \widetilde{u}, \omega\right)
$$

and

$$
\widehat{L}_{\tau, \epsilon}\left(t_{0}, x_{0}, \widetilde{u}, \omega\right)=\widehat{L}_{\tau, \epsilon}^{K}\left(t_{0}, x_{0}, \widetilde{u}, \omega\right) .
$$

These $K$ will be referred to throughout the paper.

Remark 2.4. These lemmas are not to be taken for granted. In the context of more general control theory, neither Lemma 2.1, nor Lemmas, 2.2-2.3 or even the fact that we are from the outset minimizing over controls in $L^{\infty}\left([0, T] \rightarrow \mathbb{R}^{d}\right)$, is something which is justified without strict assumptions. In the calculus of variations setting, we might more generally minimize over the set of measurable controls, $\mathcal{M}$. However there are examples in which

$$
\inf _{u \in \mathcal{M}} J(t, x, u)<\inf _{u \in \mathcal{U}} J(t, x, u) .
$$

Such examples are said to exhibit the Lavrentiev phenomenon. This shows that a careful choice of the space of minimizers is required. A particular one-dimensional example is given in [2], which shows that for the Lagrangian

$$
L(t, x, u)=\left(t^{4}-x^{6}\right)^{2}|u|^{s}+\gamma|u|^{2}
$$


on the time interval $[-1,1]$, the Lavrentiev phenomenon is exhibited for some $\gamma>0$ and $s \geq 27$, under the boundary conditions $x(-1)=k_{1}, x(1)=k_{2}$ for some $k_{1}, k_{2}$. Furthermore the Lagrangian in (2.21) satisfies every assumption in our hypothesis of Lemma 2.3 except the uniform Lipschitz continuity with respect to time, as given by the Lipschtiz constant $\|L\|_{L i p}$. If we attempted to replicate the proof of Lemma 2.3, as given in Section 3 for the Lagrangian in (2.21), it would fail for that very reason. Thus we see that the results of this paper rely delicately on the regularity assumptions.

\subsection{Continuity of the discretized control problem and value function}

In this subsection we study the error of using a stationary approximation to the control problem and prove continuity properties of $L_{\tau, \epsilon}$ and $V_{\epsilon}$. These lemmas will then be applied in the next subsection to analyse the continuity of the effective Lagrangian $\widetilde{L}$ and to show that it is almost surely constant in $\Omega$.

Lemma 2.3 is key for the following two lemmas. The first of these will be used, albeit in a different form, to show convergence of the value functions in subsection 2.5. It illustrates the error obtained if we use a stationary approximation.

Lemma 2.5. For every $\left(t_{0}, x_{0}, \widetilde{u}_{0}\right) \in[0, T] \times \mathbb{R}^{d} \times \mathbb{R}^{d}$ and $\tau>0$ such that $\tau\left|f^{*}(K)\right| \leq|\eta(K)|$, where $K=K\left(\widetilde{u}_{0}\right)$ as in Lemma 2.3, we have

$$
\left|L_{\tau, \epsilon}\left(t_{0}, x_{0}, \widetilde{u}_{0}, \omega\right)-\widehat{L}_{\tau, \epsilon}\left(t_{0}, x_{0}, \widetilde{u}_{0}, \omega\right)\right| \leq \tau^{2}\left[\|L\|_{L i p}+f^{*}(K)\|L\|_{L i p}^{K+\eta(K)}\|H\|_{L i p}^{K+\eta(K)}\right]+\tau m_{L}^{K+\eta(K)}\left(\tau f^{*}(K)\right)
$$

Proof. Take any control $u \in \widehat{\Pi}_{t_{0}, x_{0}, \tau, \widetilde{u}_{0}}$. Without loss of generality, we can assume that $\|u\|_{\infty} \leq K$. This implies that $\sup _{s \in\left[t_{0}, t_{0}+\tau\right]}\left|x(s)-x_{0}\right| \leq \tau f^{*}(K)$. Therefore we may use Assumption 1.8 and deduce that for each $s \in$ $\left[t_{0}, t_{0}+\tau\right]$ there will exist a $\bar{u} \in \mathcal{U}^{K+\eta(K)}$ such that

$$
f\left(x_{0}, \bar{u}(s)\right)=f(x(s), u(s))
$$

and

$$
|\bar{u}(s)-u(s)| \leq\|H\|_{L i p}^{K+\eta(K)}\left(\left|\tau f^{*}(K)\right|\right)
$$

this control follows the same trajectory, in that $\bar{x}(s)=x(s)$. Then

$$
\begin{aligned}
& \mid \int_{t_{0}}^{t_{0}+\tau} L\left(t_{0}, x_{0}, \frac{x(s)}{\epsilon}, \bar{u}(s), \omega\right) \mathrm{d} s-\int_{t_{0}}^{t_{0}+\tau} L\left(s, x(s), \frac{x(s)}{\epsilon}, u(s), \omega\right) \mathrm{d} s \mid \\
& \leq \tau^{2}\left[\|L\|_{L i p}+f^{*}(K)\|L\|_{L i p}^{K+\eta(K)}\|H\|_{L i p}^{K+\eta(K)}\right]+\tau m_{L}^{K+\eta(K)}\left(\tau f^{*}(K)\right)
\end{aligned}
$$

as the control $u(\cdot)$ was arbitrary, this proves that

$$
\begin{array}{r}
\widehat{L}_{\tau, \epsilon}\left(t_{0} \cdot x_{0}, \widetilde{u}, \omega\right)+\tau^{2}\left[\|L\|_{L i p}+f^{*}(K)\|L\|_{L i p}^{K+\eta(K)}\|H\|_{L i p}^{K+\eta(K)}\right]+\tau m_{L}^{K+\eta(K)}\left(\tau f^{*}(K)\right) \\
\geq L_{\tau, \epsilon}\left(t_{0} \cdot x_{0}, \widetilde{u}_{0}, \omega\right) .
\end{array}
$$

The converse inequality is proved in the same fashion and this completes the proof.

We turn our attention to obtain a modulus of continuity on $L_{\tau, \epsilon}$. 
Lemma 2.6. Suppose $\left(t_{0}, x_{0}, y\right) \in[0, T] \times \mathbb{R}^{d} \times \mathbb{R}^{d}$, and $\epsilon, \tau_{1}, \tau_{2}$ are strictly positive and $\left(\widetilde{u}_{1}, \widetilde{u}_{2}\right) \in \mathbb{R}^{d} \times \mathbb{R}^{d}$ are such that $\tau>\delta^{-1}\left(\left|\tau_{1} f\left(x_{0}, \widetilde{u}_{1}\right)-\tau_{2} f\left(x_{0}, \widetilde{u}_{2}\right)\right|\right)+\epsilon y$. Then define

$$
\begin{aligned}
& \beta_{1}=\frac{\tau_{1}}{\tau_{2}-\delta^{-1}\left(\left|\tau_{1} f\left(x_{0}, \widetilde{u}_{1}\right)-\tau_{2} f\left(x_{0}, \widetilde{u}_{2}\right)\right|+\epsilon y\right)}, \\
& \beta_{2}=\frac{\tau_{2}}{\tau_{1}-\delta^{-1}\left(\left|\tau_{2} f\left(x_{0}, \widetilde{u}_{2}\right)-\tau_{1} f\left(x_{0}, \widetilde{u}_{1}\right)\right|+\epsilon y\right)},
\end{aligned}
$$

and

$$
\begin{gathered}
\beta^{*}:=\operatorname{argmax}_{\beta_{1}, \beta_{2}}\left|\beta_{i}-1\right| \\
\beta_{*}:=\operatorname{argmax}_{\beta_{1}, \beta_{2}}\left|1 / \beta_{i}-1\right|,
\end{gathered}
$$

$\tau:=\tau_{1} \wedge \tau_{2}$ and $K:=K\left(\widetilde{u}_{1}\right) \wedge K\left(\widetilde{u}_{2}\right)$, where $K\left(\widetilde{u}_{1}\right)$ and $K\left(\widetilde{u}_{2}\right)$ are as in Lemma 2.3. Assume $\left|\beta^{*}-1\right| \leq \eta(K)$. Then for every $\omega \in \Omega$ almost surely,

$$
\begin{aligned}
& \left|L_{\tau_{1}, \epsilon}\left(t_{0}, x_{0}, \widetilde{u}_{1}, \omega\right)-L_{\tau_{2}, \epsilon}\left(t_{0}, x_{0}, \widetilde{u}_{2}, \omega\right) \circ \varphi_{y}\right| \\
& \quad \leq \tau L^{*}(K)\left|1 / \beta_{*}-1\right|+\tau\|L\|_{L i p}^{K+\eta(K)}\|H\|_{L i p}^{K+\eta(K)} f^{*}(K)\left|\beta^{*}-1\right|+\frac{L^{*}(M)}{\delta}\left(\|f\|_{L i p}^{K}\left|\widetilde{u}_{1}-\widetilde{u}_{2}\right|+\epsilon y\right) .
\end{aligned}
$$

Proof. Without any loss of generality we take $t_{0}=0$. Fix any control $u \in \Pi_{0, x_{0}, \tau_{1}, \widetilde{u}_{1}}$, and assume $\|u\|_{\infty} \leq K$. We will adopt a similar strategy of defining a control, $\bar{u}$ which traces out the same trajectory. Define $\bar{u}(s) \in \Pi_{0, x_{0}, \tau_{2}, \widetilde{u}_{2}}$ as follows. For $s \in\left[0, \tau_{1}-\epsilon \delta^{-1}|y|\right]$ define $\bar{u}$ such that

$$
f\left(x_{0}, \bar{u}(s)\right)=\delta \frac{y}{|y|}
$$

this $\delta$ is as in equation (1.43). The trajectory of $\bar{u}$, denoted $\bar{x}$, will satisfy $\bar{x}\left(\epsilon \delta^{-1}|y|\right)=x_{0}$. For $s \in\left[\epsilon \delta^{-1}|y|, \tau_{2}-\right.$ $\left.\delta^{-1}\left|\tau_{1} f\left(x_{0}, \widetilde{u}_{1}\right)-\tau_{2} f\left(x_{0}, \widetilde{u}_{2}\right)\right|\right]$ define $\bar{u}(s)$ as the solution to

$$
f\left(x_{0}, \bar{u}(s)\right)=\beta_{1} f\left(x_{0}, u(\beta s)\right) .
$$

This will reach $\tau_{1} f\left(x_{0}, \widetilde{u}_{1}\right)$ by time $\tau_{2}-\delta^{-1}\left|\tau_{1} f\left(x_{0}, \widetilde{u}_{1}\right)-\tau_{2} f\left(x_{0}, \widetilde{u}_{2}\right)\right|$, and the existence of such a control is guaranteed by Assumption 1.8 and our assumption $\left|\beta^{*}-1\right| \leq \eta(K)$. in the statement of the lemma. During this time note that

$$
\left|\bar{u}(s)-u\left(\beta_{1} s\right)\right| \leq\|H\|_{L i p}^{K+\eta(K)}\left(f^{*}(K)\left|\beta_{1}-1\right|\right) .
$$

and $\overline{x(t)}=x\left(\beta_{1} t\right)$

For $s \in\left[\tau_{2}-\delta^{-1}\left|f\left(x_{0}, \widetilde{u}_{1}\right)-f\left(x_{0}, \widetilde{u}_{2}\right)\right|, \tau_{2}\right]$ we define $\bar{u}(s)$ such that

$$
f\left(x_{0}, \bar{u}(s)\right)=\delta \frac{f\left(x_{0}, \widetilde{u}_{1}\right)-f\left(x_{0}, \widetilde{u}_{2}\right)}{\left|f\left(x_{0}, \widetilde{u}_{1}\right)-f\left(x_{0}, \widetilde{u}_{2}\right)\right|}
$$


which ensures $\bar{x}\left(\tau_{2}\right)=x_{0}+\tau_{2} f\left(x_{0}, \widetilde{u}_{2}\right)$. Then,

$$
\begin{aligned}
& \left|\int_{0}^{\tau_{1}} L\left(0, x_{0}, \frac{x(s)}{\epsilon}, u(s), \omega\right) \mathrm{d} s-\int_{0}^{\tau_{2}} L\left(0, x_{0}, \frac{\bar{x}(s)}{\epsilon}, \bar{u}(s), \omega\right) \mathrm{d} s\right| \\
& \leq\left|\int_{0}^{\tau_{1}} L\left(0, x_{0}, \frac{x(s)}{\epsilon}, u(s), \omega\right) \mathrm{d} s-\frac{1}{\beta_{1}} \int_{0}^{\tau_{1}} L\left(0, x_{0}, \frac{x(s)}{\epsilon}, \bar{u}\left(\beta_{1}^{-1} s\right), \omega\right) \mathrm{d} s\right| \\
& \quad+\int_{0}^{\epsilon \delta^{-1} y /|y|} L\left(0, x_{0}, \frac{\bar{x}(s)}{\epsilon}, \bar{u}(s), \omega\right) \mathrm{d} s+\int_{\tau_{2}-\delta^{-1}\left|\tau_{1} f\left(x_{1}, u_{1}\right)-\tau_{2} f\left(x_{2}, u_{2}\right)\right|}^{\tau_{2}} L\left(0, x_{0}, \frac{x(s)}{\epsilon}, \bar{u}(s), \omega\right) \mathrm{d} s . \\
& \quad \leq \tau L^{*}(K)\left|1 / \beta_{1}-1\right|+\tau\|L\|_{L i p}^{K+\eta(K)}\|H\|_{L i p}^{K+\eta(K)} f^{*}(K)\left|\left(\beta_{1}-1\right)\right|+\frac{L^{*}(M)}{\delta}\left(\|f\|_{L i p}^{K}\left|\widetilde{u}_{1}-\widetilde{u}_{2}\right|+\epsilon y\right)
\end{aligned}
$$

The control $u \in \Pi_{0, x_{0}, \tau_{1}, \widetilde{u}_{1}}$ was arbitrary. We can repeat the proof for an arbitrary $\hat{u} \in \widehat{\Pi}_{0, x_{0}, \tau_{2}, \widetilde{u}_{2}}$ and acquire a similar bound for involving $\beta_{2}$, and then taking the maximum over $\beta_{i}$, arrive at the conclusion.

Finally we also wish to get a uniform continuity bound on $V_{\epsilon}(t, x, \omega)$ uniform in $\epsilon$. For this proof, it similarly essential that we have effective compactness stated in Lemma 2.2.

Lemma 2.7. Let $K$ be as in as in Lemma 2.2. Then for all $\epsilon>0$, almost every $\omega$ and $x_{i} \in \mathbb{R}^{d}, t_{i} \in[0, T]$, $i \in\{1,2\}$,

$$
\begin{aligned}
\left|V_{\epsilon}\left(t_{1}, x_{1}, \omega\right)-V_{\epsilon}\left(t_{2}, x_{2}, \omega\right)\right| \leq & \frac{L^{*}(M)}{\delta}\left|x_{1}-x_{2}\right|+T|| L \|_{L i p}\left(\left|t_{1}-t_{2}\right|+\delta^{-1}\left|x_{1}-x_{2}\right|\right) \\
& +T\left(\left|t_{1}-t_{2}\right|+\delta^{-1}\left|x_{1}-x_{2}\right|\right) L^{*}(K)+m^{\psi}\left(\left|t_{1}-t_{2}\right|+\delta^{-1}\left|x_{1}-x_{2}\right|\right)
\end{aligned}
$$

Proof. The concept and argument of the proof is very similar to that of the previous lemma. If we start from $x_{1}$, we can move to position $x_{2}$ in time $\delta^{-1}\left|x_{1}-x_{2}\right|$. From here we can trace out the same trajectory. The difference that we pick up is accounted as follows

1. The first term is the cost during the time taken for the control to reach $x_{2}$. The cost of this is

$$
\int_{t_{1}}^{t_{1}-\delta^{-1}\left|x_{1}-x_{2}\right|} L\left(s, x(s), \frac{x(s)}{\epsilon}, u(s), \omega\right) \mathrm{d} s \leq \frac{L^{*}(M)}{\delta}\left|x_{1}-x_{2}\right|
$$

for some control $u(s)$ such that

$$
f(x(s), u(s))=\delta \frac{x_{2}-x_{1}}{\left|x_{2}-x_{1}\right|}
$$

2. The second term accounts for the difference in the time argument of the Lagrangian throughout the imitating control. This is bounded by $\|L\|_{L i p}\left(\left|t_{1}-t_{2}\right|+\delta^{-1}\left|x_{1}-x_{2}\right|\right)$

3. The third term follows from the fact that any control attempting to trace out another control which started at $t$, may fall short of time up to a maximum of $\left|t_{1}-t_{2}\right|+\delta^{-1}\left|x_{1}-x_{2}\right|$. However over any period of time, we can assume that the Lagrangian is bounded proportional to $L^{*}(K)$

4. The last term comes from the difference in the terminal cost, which by Assumption 1.5 is less then $m^{\psi}\left(\left|t_{1}-t_{2}\right|+\delta^{-1}\left|x_{1}-x_{2}\right|\right)$ 


\subsection{Subadditive Ergodic theorem and effective Lagrangian}

In this subsection we state a continuous parameter version of the sub-additive ergodic theorem to deduce (1.19) almost surely and define the effective Lagrangian.

Define the set $Q_{c}=\left\{(a, b) \in \mathbb{R}^{2}: 0 \leq a<b\right\}$. Let $F=\left\{F_{a, b}:(a, b) \in Q_{c}\right\}$ be a family of integrable real valued functions on our probability space $(\Omega, \mathcal{F}, P)$. Let $\left\{\widetilde{\varphi}_{r}: r \geq 0\right\}$ be a family of measure preserving mappings acting on $\Omega$. The family $F_{a, b}$ is called a continuous sub-additive process if the conditions

(C1) $F_{a, b} \circ \tilde{\varphi}_{r}=F_{a+r, b+r} \quad\left((a, b) \in Q_{c}, l \geq 0\right)$

(C2) $F_{a, l} \leq F_{a, b}+F_{b, l} \quad\left((a, l),(b, l) \in Q_{c}\right)$

(C3) $\mathbb{E}\left(F_{0, b}\right) \geq \gamma b$ for some $\gamma>-\infty$

are satisfied and $F_{a, b}(\omega)$ is a measurable map of $Q_{c} \times \Omega \rightarrow \mathbb{R}$ with respect to the product $\sigma$-algebra in $Q_{c} \times \Omega$. The expectation is with respect to the measure $P$.

For the statement and proof of the following proposition we refer to [13].

Proposition 2.8. Suppose that $\left\{F_{a, b}\right\}_{(a, b) \in Q_{c}}$ satisfies (C1)-(C3), is continuous in $a, b$ and there exists a $K(a, b)$ such that $\left|F_{a, b}(\omega)\right| \leq K(a, b)$ a.s. ${ }^{1}$ Then

$$
\lim _{b \rightarrow \infty} b^{-1} F_{0, b}
$$

exists a.s. and is $\widetilde{\varphi}_{r}$ invariant for all $r \geq 0$.

To apply the sub-additive ergodic theorem to $L_{\tau, \epsilon}$, we need to reintepret our problem as "long term averages."

We will fix a a $\left(t_{0}, x_{0}, \widetilde{u}_{0}\right) \in[0, T] \times \mathbb{R}^{d} \times \mathbb{R}^{d}$ and $\tau>0$. For $u(s) \in \Pi_{t_{0}, x_{0}, \tau, \widetilde{u}_{0}}$, we have that by substitution $\widetilde{s}=s / \epsilon$

$$
\frac{x(t+\tau)}{\epsilon}=\frac{x_{0}}{\epsilon}+\int_{t / \epsilon}^{(t+\tau) / \epsilon} f\left(x_{0}, u(\epsilon \widetilde{s})\right) \mathrm{d} \widetilde{s}=: \widetilde{x}(t+\tau) .
$$

After the same substitution $\widetilde{s}$ our object of interest is equivalent to

$$
L_{\tau, \epsilon}\left(t_{0}, x_{0}, \widetilde{u}_{0}, \omega\right)=\inf _{u \in \Pi_{t, x, \tau / \epsilon, \tilde{u}_{0} / \epsilon}} \epsilon \int_{t / \epsilon}^{(t+\tau) / \epsilon} L\left(t_{0}, x_{0}, \widetilde{x}(\widetilde{s}), u(\widetilde{s}), \omega\right) \mathrm{d} \widetilde{s}
$$

The distinguishment between $(\widetilde{x}(s), \widetilde{s})$ and $(x(s), s)$ is superficial, we shall henceforth write $(x(s), s)$. For our purpose, we then define $F_{a, b}$ as

$$
F_{a, b}:=\inf _{u \in \Pi_{t_{0}, x_{0}+a f\left(x_{0}, \tilde{u}_{0}\right), b-a, \tilde{u}_{0}}} \int_{t_{0}}^{t_{0}+b-a} L\left(t_{0}, x_{0}, x(s), u(s), \omega\right) \mathrm{d} s .
$$

Then using equation (2.42), for each $\epsilon>0$ this is equivalent to

$$
\frac{1}{\epsilon} L_{\epsilon(b-a), \epsilon}\left(\epsilon t_{0}, x_{0}+\epsilon a f\left(x_{0}, \widetilde{u}_{0}\right), \widetilde{u}_{0}, \omega\right)
$$

\footnotetext{
${ }^{1}$ In the formulation given in [13], the conditions are weaker. $F_{a, b}$ is assumed to be separable and has integrable oscillations. We refer the reader to [13] for further details.
} 
The family of measure preserving transformations $\left\{\widetilde{\varphi}_{r}: r \geq 0\right\}$ is then defined as $\varphi_{r f\left(x_{0}, \widetilde{u}_{0}\right)}$ - the ergodic measure preserving transformations from Assumption 1.1. Therefore

$$
\begin{aligned}
F_{a, b} \circ \widetilde{\varphi}_{r} & =\inf _{u \in \Pi_{t_{0}, x_{0}+a f\left(x_{0}, \tilde{u}_{0}\right), b-a, u}} \int_{t_{0}}^{t_{0}+b-a} L\left(t_{0}, x_{0}, x(s), u(s), \varphi_{r f\left(x_{0}, \widetilde{u}_{0}\right)} \omega\right) \mathrm{d} s \\
& =\inf _{u \in \Pi_{t_{0}, x_{0}+a f\left(x_{0}, \tilde{u}_{0}\right), b-a, \tilde{u}_{0}}} \int_{t_{0}}^{t_{0}+b-a} L\left(t_{0}, x_{0}, x(s)+r f\left(x_{0}, \widetilde{u}_{0}\right), u(s), \omega\right) \mathrm{d} s \\
& =\inf _{u \in \Pi_{t_{0}, x_{0}+(a+r) f\left(x_{0}, \tilde{u}_{0}\right), b-a, \tilde{u}_{0}}} \int_{t_{0}}^{t_{0}+b-a} L\left(t_{0}, x_{0}, x(s), u(s), \omega\right) \mathrm{d} s \\
& =F_{a+r, b+r}
\end{aligned}
$$

and so $(\mathrm{C} 1)$ is satisfied. Sub-additivity $(\mathrm{C} 2)$ is a consequence of the fact that any path from $x_{0}$ to $x_{0}+a f\left(x_{0}, \widetilde{u}_{0}\right)$ in time $[0, a]$, and then a path from $x_{0}+a f\left(x_{0}, \widetilde{u}_{0}\right)$ to $x_{0}+b f\left(x_{0}, \widetilde{u}_{0}\right)$ in time $[a, b]$, can be joined to form a path from $x_{0}$ to $b f\left(x_{0}, \widetilde{u}_{0}\right)$ in time $[0, b]$. The condition (C3) follows from the assumption that $L_{*}(0)$ is finite.

We now want to show that it satisfies the conditions of Proposition 2.8. The upper bound $K(a, b)$ can be taken as $(b-a) L^{*}\left(K\left(\widetilde{u}_{0}\right)\right)$ where the $K\left(\widetilde{u}_{0}\right)$ is as in equation (2.18). Hence we only need the following continuity lemma.

Lemma 2.9. For positive real numbers $b_{1}, b_{2}, a_{1}, a_{2}$ such that $\left|b_{1}-b_{2}\right|,\left|a_{1}-a_{2}\right|$ are sufficiently small there exists a constant $C=C(k)$ such that if $\left|b_{1}-a_{1}\right| \wedge\left|b_{2}-a_{2}\right|>k$

$$
\left|F_{a_{1}, b_{1}}-F_{a_{2}, b_{2}} \circ \varphi_{y}\right| \leq C\left[\left|a_{1}-a_{2}\right|+\left|b_{1}-b_{2}\right|+|y|\right]
$$

Proof. The proof occurs through using Lemma 2.6 in the special case where $\widetilde{u}_{1}=\widetilde{u}_{2}=\widetilde{u}_{0}$ and the equation (2.44). Doing so we observe that, for each $\epsilon>0$,

$$
\begin{aligned}
& \left|F_{0, b_{1}} \circ \varphi_{y}-F_{0, b_{2}}\right|=\frac{1}{\epsilon}\left|L_{\epsilon b_{1}, \epsilon}\left(\epsilon t_{0}, x_{0}, \widetilde{u}_{0}, \omega\right)-L_{\epsilon b_{2}, \epsilon}\left(\epsilon t_{0}, x_{0}, \widetilde{u}_{0}, \omega\right)\right| \\
& \leq\left|b_{1}-b_{2}\right|\left(L^{*}(K)\left|1 / \beta_{*}-1\right|+\|L\|_{L i p}^{K+\eta(K)}\|H\|_{L i p}^{K+\eta(K)} f^{*}(K)\left|\left(\beta^{*}-1\right)\right|\right)+\frac{L^{*}(M)}{\delta}|y|
\end{aligned}
$$

provided that $\left|b_{1}-b_{2}\right|$ and $y$ are sufficiently small so that the hypothesis of Lemma 2.6 are satisfied. $\beta^{*}$ and $\beta_{*}$ are as in Lemma 2.6. Note $\beta^{*}$ and $\beta_{*}$ diverges/becomes indeterminant for small values of $b_{1}, b_{2}$ and hence the Lipschitz constant $C(k)$ depends on the parameter $k$. This is not an issue as we will be interested in the regime when $b_{1}, b_{2}$ are large.

This proves Lipschitz continuity of the object $F_{a, b} \circ \varphi_{y}$ in the $y$ variable and second parameter. The continuity of the first parameter is proved similar to the analysis in Lemma 2.6.

We may now utilize the Proposition 2.8 to deduce on a set $\Omega_{0} \subseteq \Omega$ with $P\left(\Omega_{0}\right)=1$ there exists an $\widetilde{L}_{\tau}\left(t_{0}, x_{0}, \widetilde{u}_{0}, \omega\right)$ such that

$$
\lim _{\epsilon \rightarrow 0} \epsilon F_{0, \tau / \epsilon}=\lim _{\epsilon \rightarrow 0} L_{\tau, \epsilon}\left(t_{0}, x_{0}, \widetilde{u}_{0}, \omega\right)=\widetilde{L}_{\tau}\left(t_{0}, x_{0}, \widetilde{u}_{0}, \omega\right)=\tau \widetilde{L}_{1}\left(t_{0}, x_{0}, \widetilde{u}_{0}, \omega\right)
$$

for all $\omega \in \Omega_{0}$. The linear relationship of $\widetilde{L}$ with respect its argument $\tau$ will become important later.

Take any $y \in \mathbb{R}^{d}$ and observe that, from (2.50),

$$
\lim _{\epsilon \rightarrow 0} \epsilon\left|F_{0, \tau / \epsilon} \circ \varphi_{y}-F_{0, \tau / \epsilon}\right| \leq \lim _{\epsilon \rightarrow 0} \epsilon C|y|=0 .
$$


Hence it follows that $\widetilde{L}_{\tau}\left(t_{0}, x_{0}, \widetilde{u}_{0}, \omega\right)$ is $\varphi_{y}$ invariant. Therefore by ergodicity, $\widetilde{L}_{\tau}\left(t_{0}, x_{0}, \widetilde{u}_{0}, \omega\right)$ is almost surely constant in $\Omega$.

We then extend this to every rational number and take the countable intersection to conclude the following lemma.

Lemma 2.10. There is a function $\widetilde{L}_{\tau}$ such that, for all $\left(t_{0}, x_{0}, \widetilde{u}_{0}\right) \in\left([0, T] \cap \mathbb{Q}^{\prime} \times \mathbb{Q}^{d} \times \mathbb{Q}^{d}\right.$, we have an event $\Omega^{\prime} \subset \Omega$ with $P\left(\Omega^{\prime}\right)=1$ such that

$$
\lim _{\epsilon \rightarrow 0} L_{\tau, \epsilon}\left(t_{0}, x_{0}, \widetilde{u}_{0}, \omega\right)=\widetilde{L}_{\tau}\left(t_{0}, x_{0}, \widetilde{u}_{0}\right) \text { on } \Omega^{\prime}
$$

For a control $\widetilde{u}=\left\{\widetilde{u}_{i}\right\}_{i=0}^{N-1} \in \mathcal{U}_{\tau}$ we define the cost function

$$
J_{\tau}\left(t_{0}, x_{0}, \widetilde{u}\right)=\sum_{i=0}^{N-1} \widetilde{L}_{\tau}\left(t_{i}, x_{i}, \widetilde{u}_{i}\right)
$$

and value function

$$
\widetilde{V}_{\tau}\left(t_{0}, x_{0}\right)=\inf _{\widetilde{u} \in \mathcal{U}_{\tau}} J_{\tau}(t, x, \widetilde{u})
$$

Recalling the linear relationship of $\widetilde{L}_{\tau}$ with respect to $\tau$, we define the effective Lagrangian

$$
L\left(t_{0}, x_{0}, \widetilde{u}_{0}\right):=\frac{1}{\tau} \widetilde{L}_{\tau}\left(t_{0}, x_{0}, \widetilde{u}_{0}\right)=\widetilde{L}_{\tau}\left(t_{0}, x_{0}, \widetilde{u}_{0}\right)
$$

for any $\tau>0$.

Lemma 2.11. For each $R>0, \widetilde{L}(\cdot, \cdot, \cdot)$ is uniformly continuous on $([0, T] \cap \mathbb{Q}) \times \mathbb{Q}^{d} \times\left(\mathbb{Q}^{d} \cap U^{R}\right)$ and hence can be extended to all real numbers.

Proof. In this proof we always take $\left(t_{i}, x_{i}, u_{i}\right) \in([0, T] \cap \mathbb{Q}) \times \mathbb{Q}^{d} \times\left(\mathbb{Q}^{d} \cap U^{R}\right)$. Employing Lemma 2.6 and the $\beta_{i}$ therein, for any fixed $\tau$ we will have

$$
\beta:=\beta_{1}=\beta_{2}=\frac{1}{1-\delta^{-1}\left|f\left(x_{0}, u_{2}\right)-f\left(x_{0}, u_{2}\right)\right|} .
$$

This quantity is also $\tau$ invariant, and thus, by Lemma 2.6 we immediately obtain a uniform modulus of continuity in the control variable on $U^{R}$ and in $\tau$.

The temporal and spatial modulus of continuity are inherited from the original Lagrangian, after taking $\epsilon \rightarrow 0$. Specifically, we have, for almost all $\omega \in \Omega$

$$
\begin{aligned}
=\left|\widetilde{L}_{1}\left(t_{1}, x_{1}, \widetilde{u}_{i}\right)-\widetilde{L}_{1}\left(t_{2}, x_{2}, \widetilde{u}_{i}\right)\right| & \\
& \leq \lim _{\epsilon \rightarrow 0}\left|L_{1, \epsilon}\left(t_{2}, x_{2}, \widetilde{u}_{i}, \omega\right)-L_{1, \epsilon}\left(t_{2}, x_{2}, \widetilde{u}_{i}, \varphi_{x_{1}-x_{2}} \omega\right)\right|+\|L\|_{L i p}\left(\left|t_{1}-t_{2}\right|\right)+m_{L}^{\left|\widetilde{u}_{i}\right|}\left(\left|x_{1}-x_{2}\right|\right)
\end{aligned}
$$

However as the $\widetilde{L}_{\tau}$ is almost surely constant on $\Omega$, then

$$
\lim _{\epsilon \rightarrow 0}\left|L_{1}\left(t_{2}, x_{2}, \widetilde{u}_{i}, \omega\right)-L_{1}\left(t_{2}, x_{2}, \widetilde{u}_{i}, \varphi_{x_{1}-x_{2}} \omega\right)\right|=0 \text { a.s. }
$$


Therefore

$$
\left|\widetilde{L}\left(t_{1}, x_{1}, \widetilde{u}_{i}\right)-\widetilde{L}\left(t_{2}, x_{2}, \widetilde{u}_{i}\right)\right| \leq\|L\|_{L i p}\left(\left|t_{1}-t_{2}\right|\right)+m_{L}^{\left|\widetilde{u}_{i}\right|}\left(\left|x_{1}-x_{2}\right|\right)
$$

This shows that $\widetilde{L}$ is Lipschitz continuous on $\mathcal{U}^{K}$ for each $K>0$ and is Lipschitz continuous in the temporal variable. We will denote these Lipschitz moduli of continuity as $\|\widetilde{L}\|_{L i p}^{K}$ and $\| \widetilde{\|}_{L i p}$ respectively.

Having extended the effective Lagrangian to all of $\mathbb{R}^{d}$ we can now define the homogenized control problem. For some control $u \in \mathcal{U}$, let $x(\cdot)$ be given by the state dynamics as in (1.3). We define the cost functional as

$$
\widetilde{J}(t, x, u):=\int_{t}^{T} \widetilde{L}(s, x(s), u(s)) \mathrm{d} s
$$

for $(t, x, \omega) \in[0, T] \times \mathbb{R}^{d} \times \Omega$ and the value function

$$
\widetilde{V}(t, x):=\inf _{u \in \mathcal{U}} \widetilde{J}(t, x, u)
$$

\subsection{Coercivity of the effective Lagrangian}

Lemma 2.12. For all $\left(t_{0}, x_{0}, \widetilde{u}_{0}\right) \in[0, T] \times \mathbb{R}^{d} \times \mathbb{R}^{d}$ and $\epsilon, \tau>0$ we have that

$$
L_{\tau, \epsilon}\left(t_{0}, x_{0}, \widetilde{u}_{0}\right) \geq \tau L_{*}\left(\widetilde{u}_{0}\right)
$$

Proof. Without loss of generality, take $\tau=1$ and suppose that $\tau_{1}, \tau_{2} \geq 0$ such that $\tau_{1}+\tau_{2}=1$.

Suppose that $\widetilde{u}_{1}$ and $\widetilde{u}_{2}$ are such that

$$
\tau_{1} f\left(x_{0}, \widetilde{u}_{1}\right)+\tau_{2} f\left(x_{0}, \widetilde{u}_{2}\right)=f\left(x_{0}, \widetilde{u}_{0}\right)
$$

then the essence of the proof is showing that this implies that

$$
\tau_{1} L_{*}\left(\widetilde{u}_{1}\right)+\tau_{2} L_{*}\left(\widetilde{u}_{2}\right) \geq L_{*}\left(\widetilde{u}_{0}\right) .
$$

The argument is as follows. We have from Assumption 1.8 that

$$
\tau_{1} L_{*}\left(\widetilde{u}_{1}\right)-\tau_{1} L_{*}\left(\widetilde{u}_{0}\right) \geq \tau_{1} \Theta\left(\widetilde{u}_{2}\right) \cdot\left[f\left(x, \widetilde{u}_{1}\right)-f\left(x, \widetilde{u}_{0}\right)\right]
$$

and

$$
\tau_{2} L_{*}\left(\widetilde{u}_{2}\right)-\tau_{2} L_{*}(u) \geq \tau_{2} \Theta\left(\widetilde{u}_{2}\right) \cdot\left[f\left(x, \widetilde{u}_{2}\right)-f\left(x, \widetilde{u}_{0}\right)\right] .
$$

Adding these gives the inequality

$$
\tau_{1} L_{*}\left(\widetilde{u}_{1}\right)+\tau_{2} L_{*}\left(\widetilde{u}_{2}\right) \geq L_{*}\left(\widetilde{u}_{0}\right) .
$$

Iterating and passing to limits we can conclude for any $u \in \Pi_{t_{0}, x_{0}, \tau, \widetilde{u}_{0}}$,

$$
\int_{t_{0}}^{t_{0}+\tau} L\left(t_{0}, x_{0}, \frac{x(s)}{\epsilon}, u(s), \omega\right) \mathrm{d} s \geq \int_{t_{0}}^{t_{0}+\tau} L_{*}(u(s)) \mathrm{d} s \geq L_{*}\left(\widetilde{u}_{0}\right) .
$$


By taking $\epsilon \rightarrow 0$ we can immediately conclude from this Lemma that $\widetilde{L}_{\tau}(t, x, u) \geq \tau L_{*}(u)$ for every $(t, x, u) \in$ $[0, T] \times \mathbb{R}^{d} \times \mathbb{R}^{d}$ and $\tau>0$. This lemma gives us precisely the same estimates on $\widetilde{L}, L_{\tau, \epsilon}$ as $L$, and so we can prove the effective boundedness for the homogenized control problems.

Lemma 2.13. There exists an $K>0$ such that for all $t \in[0, T], x \in \mathbb{R}^{d}$.

$$
\widetilde{V}^{K}(t, x)=\widetilde{V}(t, x),
$$

and

$$
\widetilde{V}_{\tau}^{K}(t, x)=\widetilde{V}_{\tau}(t, x)
$$

In order to understand the nature of this $\Theta$, consider the case where $f$ and $L_{*}(\cdot)$ is continuously differentiable and that the Jacobian of $f$ is invertible at every point. Then similar to lemmas in classic convex analysis we can prove that the function $\Theta(\cdot)$ is the gradient of $L_{*}(\cdot)$ multiplied by the inverse of the Jacobian. Given the conclusion that

$$
(1-\tau) L_{*}\left(\widetilde{u}_{1}\right)+\tau L_{*}\left(\widetilde{u}_{2}\right) \geq L_{*}\left(\widetilde{u}_{0}\right)
$$

for $\tau \in[0,1]$ and $\widetilde{u}_{1}, \widetilde{u}_{2}$ such that $(1-\tau) f\left(x_{0}, \widetilde{u}_{1}\right)+\tau f\left(x_{0}, \widetilde{u}_{2}\right)=f\left(x_{0}, \widetilde{u}_{0}\right)$, we can make a brief calculation to deduce that

$$
L_{*}\left(\widetilde{u}_{2}\right)-L_{*}\left(\widetilde{u}_{1}\right) \geq \frac{L_{*}\left(\widetilde{u}_{1}\right)-L_{*}\left(\widetilde{u}_{1}+\tau J^{-1}\left(x_{0}, \widetilde{u}_{1}\right)\left[f\left(x_{0}, \widetilde{u}_{1}\right)-f\left(x_{0}, \widetilde{u}_{2}\right)+o(\tau)\right]\right)}{\tau}
$$

Taking $\tau \rightarrow 0$ implies that

$$
L_{*}\left(\widetilde{u}_{2}\right)-L_{*}\left(\widetilde{u}_{1}\right) \geq \nabla L_{*}\left(\widetilde{u}_{1}\right) \cdot\left[J^{-1}\left(x_{0}, \widetilde{u}_{1}\right)\left(f\left(x_{0}, \widetilde{u}_{2}\right)-f\left(x_{0}, \widetilde{u}_{1}\right)\right)\right]
$$

Thus we can essentially calculate the function $\Theta(\cdot)$.

\subsection{Convergence of the value functions}

In this subsection we prove Theorem 1.10.

Lemma 2.14. For every $t \in[0, T], x \in \mathbb{R}^{d}$ and almost all $\omega \in \Omega$

$$
V_{\tau, \epsilon}(t, x, \omega) \rightarrow V_{\epsilon}(t, x, \omega)
$$

uniformly in $\epsilon$ as $\tau \rightarrow 0$.

Proof. Similar to the preceding sections, the argument is based on imitating the trajectory and showing that the difference in cost is small.

Let $\kappa>0$ be arbitrary, take a $u \in \mathcal{U}$ such that

$$
J_{\epsilon}(t, x, u, \omega) \leq V_{\epsilon}(t, x, \omega)+\frac{\kappa}{2} .
$$


Using Lemma 2.2 we can without of generality assume that $\|u\|_{\infty} \leq K$. Define an associated approximation $\widetilde{u}$ in $\mathcal{U}_{\tau}$ by iterative construction. Set $t_{0}=t$,

$$
t_{1}=\left(t_{0}+\tau\right) \wedge T
$$

and $x_{0}=x\left(t_{0}\right)=x$. We will show that there is a $\widetilde{u}_{1} \in U^{K}$ such that

$$
f\left(x_{0}, \widetilde{u}_{1}\right)=\frac{x\left(t_{1}\right)-x}{\tau} .
$$

We can, without any loss of generality, suppose that the control is a step function taking values $u_{k}$ at times $\left[\bar{t}_{k}, \bar{t}_{k+1}\right)$ (see [19] Prop. 22, P. 69). Then

$$
x\left(t_{1}\right)-x\left(t_{0}\right)=\sum_{k=1}^{N} \int_{\bar{t}_{k}}^{\bar{t}_{k+1}} f\left(x(s), u_{k}\right) \mathrm{d} s .
$$

So, stipulating that $\tau>0$ is sufficiently small so that $\eta(K) \leq \tau f^{*}(K)$, we can use Assumption 1.8 to deduce that there exist $\bar{u}_{k}$ such that $f\left(x(s), u_{k}\right)=f\left(x_{0}, \bar{u}_{k}\right)$ at each $\left[\bar{t}_{k}, \bar{t}_{k+1}\right]$.

$$
x\left(t_{1}\right)-x\left(t_{0}\right)=\sum_{k=1}^{N} \int_{\bar{t}_{k}}^{\bar{t}_{k+1}} f\left(x_{0}, \bar{u}_{k}\right) \mathrm{d} s=\sum_{k=1}^{N}\left[\bar{t}_{k+1}-\bar{t}_{k}\right] f\left(x_{0}, u_{k}\right) .
$$

Divide both sides by $\tau$ and use that fact that the sum of $\bar{t}_{k}-\bar{t}_{k+1}$ is $\tau$ and $f\left(x_{0}, U^{K}\right)$ is a convex set to derive the existence of a $\widetilde{u}_{1}$ such that

$$
f\left(x_{0}, \widetilde{u}_{1}\right)=\frac{x\left(t_{1}\right)-x}{\tau} .
$$

We iterate this procedure to define

$$
t_{i}:=\left(t_{i-1}+\tau\right) \wedge T
$$

Similarly for each $i$ there exists $\widetilde{u}_{i}$ such that

$$
f\left(x_{i}, \widetilde{u}_{i}\right)=\frac{x\left(t_{i}\right)-x_{i-1}}{\tau}
$$

where we define $x_{i}=x\left(t_{i}\right)$. We terminate on the first $N$ such that $t_{N}=T$. These $\widetilde{u}_{i}$ then define a $\widetilde{u}=\left\{\widetilde{u}_{i}\right\}_{i=0}^{N-1}$ in $\mathcal{U}_{\tau}$.

By Lemma 2.5, in light of the fact that $t_{i+1}-t_{i} \leq \tau$,

$$
\begin{aligned}
L_{\tau, \epsilon}\left(t_{i}, x_{i}, \widetilde{u}_{i}, \omega\right)-\tau^{2}\left[\|L\|_{L i p}+f^{*}(K)\|L\|_{L i p}^{K+\eta(K)}\|H\|_{L i p}^{K+\eta(K)}\right]-\tau m_{L}^{K+\eta(K)}\left(\tau f^{*}(K)\right) \\
\quad \leq \widehat{L}_{\tau, \epsilon}\left(t_{i}, x_{i}, \widetilde{u}_{i}\right) \leq \int_{t_{i}}^{t_{i+1}} L\left(s, x(s), \frac{x(s)}{\epsilon}, u(s), \omega\right) \mathrm{d} s .
\end{aligned}
$$

Summing over $i$ we conclude

$$
J_{\tau, \epsilon}\left(t_{0}, x_{0}, \widetilde{u}, \omega\right)-T \tau\left[\|L\|_{L i p}+f^{*}(K)\|L\|_{L i p}^{K+\eta(K)}\|H\|_{L i p}^{K+\eta(K)}\right]-T m_{L}^{K+\eta(K)}\left(\tau f^{*}(K)\right)
$$




$$
\leq V_{\epsilon}\left(t_{0}, x_{0}, \omega\right)+\kappa .
$$

Conversely for a given $\kappa>0$, suppose $\widetilde{u}=\left\{\widetilde{u}_{i}\right\}_{i=1}^{N} \in \mathcal{U}_{\tau}$ is such that

$$
J_{\tau, \epsilon}(t, x, \widetilde{u}, \omega) \leq V_{\tau, \epsilon}(t, x, \omega)+\frac{\kappa}{2} .
$$

We know by Lemma 2.2 that we can without loss of generality assume that $\left|\widetilde{u}_{i}\right| \leq K$ for every $i$, uniformly in $\tau, \epsilon$.

Let $u_{i}$ be a sequence of controls such that, for each $i \leq N$,

$$
\int_{t_{i}}^{t_{i+1}} L\left(t_{i}, x_{i}, \frac{x(s)}{\epsilon}, u_{i}(s), \omega\right) \mathrm{d} s \leq L_{\tau, \epsilon}\left(t_{i}, x_{i}, \widetilde{u}_{i}\right)+\frac{\kappa}{2}\left(t_{i+1}-t_{i}\right)
$$

We know by Lemma 2.2 that for every $\widetilde{u}_{i}$ there is a $\widetilde{K}\left(\widetilde{u}_{i}\right)$ such that we may assume $\left\|u_{i}\right\|_{\infty} \leq \widetilde{K}\left(\widetilde{u}_{i}\right)$. However as $\sup _{i \leq N}\left|\widetilde{u}_{i}\right| \leq K$ then we may strengthen this to $\sup _{i \leq N}\left\|u_{i}\right\|_{\infty} \leq \widetilde{K}(K)=: \widehat{K}$.

Hence, by Lemma 2.5,

$$
\begin{aligned}
\widetilde{L}_{\tau, \epsilon}\left(t_{i}, x_{i}, u_{i}, \omega\right)-\tau^{2}\left[\|L\|_{L i p}+f^{*}(K)\|L\|_{L i p}^{\widehat{K}+\eta(\widehat{K})}\|H\|_{L i p}^{\widehat{K}+\eta(\widehat{K})}\right]-\tau m_{L}^{\widehat{K}+\eta(\widehat{K})}\left(\tau f^{*}(\widehat{K}+\eta(\widehat{K}))\right)+\frac{\kappa}{2} \\
\geq \widehat{L}_{\tau, \epsilon}\left(t_{i}, x_{i}, \widetilde{u}_{i} \omega\right) \geq \int_{t_{i}}^{t_{i+1}} L\left(s, x(s), \frac{x(s)}{\epsilon}, u(s), \omega\right) \mathrm{d} s .
\end{aligned}
$$

As $\kappa>0$ was arbitrary, summing up over $i$ we get the converse of (2.85). Thus taking $K^{*}:=\widehat{K} \wedge K$ such that

$$
\begin{aligned}
& \left|V_{\tau, \epsilon}(t, x, \omega)-V_{\epsilon}(t, x, \omega)\right| \\
& \leq T \tau\left[\|L\|_{L i p}+f^{*}\left(K^{*}\right)\|L\|_{L i p}^{K^{*}+\eta\left(K^{*}\right)}\|H\|_{L i p}^{K^{*}+\eta\left(K^{*}\right)}\right]+T m_{L}^{K^{*}+\eta\left(K^{*}\right)}\left(\tau f^{*}\left(K^{*}\right)\right)+\kappa .
\end{aligned}
$$

Observing the RHS is independent of $\epsilon$ completes the proof.

We now show that the discrete homogenized control problem converges to the continuous homogenized problem as $\tau \rightarrow 0$.

Lemma 2.15. For each $t \in[0, T], x \in \mathbb{R}^{d}$

$$
\lim _{\tau \rightarrow 0} \widetilde{V}_{\tau}(t, x)=\widetilde{V}(t, x) .
$$

Proof. Fix a $\tau>0$. Let $\widetilde{u}=\left\{\widetilde{u}_{i}\right\}_{i=1}^{N} \in \mathcal{U}_{\tau}$, with associated time intervals $\left[t_{i}, t_{i+1}\right)$ and spatial points $x_{i}$. As usual we can use Lemma 2.2 to assume that for some $K$ we have $\left|\widetilde{u}_{i}\right| \leq K$ for every $i$. We can define a $u \in \mathcal{S}$ such that $f\left(x_{i}, \widetilde{u}_{i}\right)=f(x(s), u(s))$ on $s \in\left[t_{i}, t_{i+1}\right)$. Using Assumption 1.8 this will satisfy $\left|\widetilde{u}_{i}-u(s)\right| \leq \tau f^{*}(K)\|H\|_{L i p}^{K}$. Then we have that

$$
\widetilde{J}(t, x, u)=\int_{t}^{T} \widetilde{L}(s, x(s), u(s))=\sum_{i=1}^{N} \int_{t_{i}}^{t_{i+1}} \widetilde{L}(s, x(s), u(s)) \mathrm{d} s .
$$

Because

$$
\left|\widetilde{L}(s, x(s), u(s))-\widetilde{L}\left(t_{i}, x_{i}, \widetilde{u}_{i}\right)\right| \leq \tau\|\widetilde{L}\|_{L i p}^{K+\eta(K)}\|H\|_{L i p}^{K+\eta(K)}+\tau\|L\|_{L i p}+m_{L}^{K+\eta(K)}\left(\tau f^{*}(K)\right)
$$


and by definition of $\widetilde{L}$ in $(2.56)$ we have

$$
\int_{t_{i}}^{t_{i+1}} \widetilde{L}\left(t_{i}, x_{i}, \widetilde{u}_{i}\right) \mathrm{d} s=\tau \widetilde{L}\left(t_{i}, x_{i}, \widetilde{u}_{i}\right) \mathrm{d} s=\widetilde{L}_{\tau}\left(t_{i}, x_{i}, \widetilde{u}_{i}\right) .
$$

Then,

$$
\left|\widetilde{J}_{\tau}(t, x, \widetilde{u})-\widetilde{J}(t, x, u)\right| \leq \tau\|\widetilde{L}\|_{L i p}^{K+\eta(K)}\|H\|_{L i p}^{K+\eta(K)}+\tau\|L\|_{L i p}+m_{L}^{K+\eta(K)}\left(\tau f^{*}(K)\right)
$$

In particular if given a $\kappa>0$ and a control $\widetilde{u}$ such that

$$
\widetilde{J}_{\tau}(t, x, u) \leq \widetilde{V}_{\tau}(t, x)+\kappa
$$

then this proves that

$$
\widetilde{V}_{\tau}(t, x)+\tau\|\widetilde{L}\|_{L i p}^{K+\eta(K)}\|H\|_{L i p}^{K+\eta(K)}+\tau\|L\|_{L i p}+m_{L}^{K+\eta(K)}\left(\tau f^{*}(K)\right)+\kappa \geq \widetilde{V}(t, x) .
$$

Conversely, fix a control $u \in \mathcal{U}$. Without loss of generality we may assume that $u \in \mathcal{S}$. We now define a $\widetilde{u} \in \mathcal{U}_{\tau}$ precisely as in the previous lemma through equations (2.82), (2.83) and denote the sequence of controls in $\mathcal{U}$ as $\widetilde{u}_{i}$ and it's time partitions as $\left[\widetilde{t}_{j}, \widetilde{t}_{j+1}\right]$. If we have for some $j, i$ that $\left[\widetilde{t}_{j}, \widetilde{t}_{j+1}\right] \subset\left[t_{i}, t_{i+1}\right]$, then it follows that $\left|\widetilde{u}_{i}-u(s)\right| \leq \tau f(K)\|H\|_{L i p}^{K}$ as $f\left(x_{i}, u_{i}\right)=f(x(s), u(s))$ for $s \in\left[\widetilde{t}_{j}, \widetilde{t}_{j+1}\right]$, which implies that during this time

$$
\left|\widetilde{L}(s, x(s), u(s))-\widetilde{L}\left(t_{i}, x_{i}, u_{i}\right)\right| \leq \tau\|\widetilde{L}\|_{L i p}^{K}\|H\|_{L i p}^{K}+\tau\|L\|_{L i p}+m_{L}^{K+\eta(K)}\left(\tau f^{*}(K)\right)
$$

We want to argue that the vast majority of $j$ falls within such an $i$.

Let $P$ be the number of partitions of the control $u$. $P$ is independent of $\tau$. Then observe that all but $P$ of the $j$ must satisfy $\left[\widetilde{t}_{j}, \widetilde{t}_{j+1}\right] \subset\left[t_{i}, t_{i+1}\right]$ and on each $j$ we have that $\widetilde{t}_{j+1}-\widetilde{t}_{j} \leq \tau$. Then

$$
\left|\widetilde{J}(t, x, u)-\widetilde{J}_{\tau}(t, x, \widetilde{u})\right| \leq T \tau\left(\|\widetilde{L}\|_{L i p}^{K}\|H\|_{L i p}^{K}+\|L\|_{L i p}+P L^{*}(K)\right)+m_{L}^{K+\eta(K)}\left(\tau f^{*}(K)\right)
$$

In particular if we have for a given $\kappa>0$ that

$$
\widetilde{J}(t, x, u) \leq \widetilde{V}(t, x)+\kappa .
$$

Then we deduce that

$$
\widetilde{V}(t, x)+T \tau\left(\|\widetilde{L}\|_{L i p}^{K}\|H\|_{L i p}^{K}+\|L\|_{L i p}+P L^{*}(K)\right)+m_{L}^{K+\eta(K)}\left(\tau f^{*}(K)\right)+\kappa \geq \widetilde{V}_{\tau}(t, x) .
$$

We now take $\tau \rightarrow 0$, as $\kappa$ was arbitrary, this concludes the proof.

Remark 2.16. In the proofs of Lemma's 2.14 and 2.15, the terminal cost function plays no role. The discrete trajectory and continuous are always such that $x_{N}=x(T)$. This will also be true in the Lemmas 2.1 and 2.2 which will be proved later.

We can now combine this to prove the main result.

Proof of Theorem 1.1. For all $t \in[0, T], x \in \mathbb{R}^{d}$ and almost all $\omega \in \Omega$ we have

$$
\begin{aligned}
& \left|V_{\epsilon}(t, x, \omega)-\widetilde{V}(t, x)\right| \\
& \quad \leq\left|V_{\epsilon}(t, x, \omega)-V_{\tau, \epsilon}(t, x)\right|+\left|V_{\tau, \epsilon}(t, x)-\widetilde{V}_{\tau}(t, x)\right|+\left|\widetilde{V}_{\tau}(t, x)-\widetilde{V}(t, x)\right| .
\end{aligned}
$$


For the second term,

$$
\lim _{\epsilon \rightarrow 0}\left|\widetilde{V}_{\tau, \epsilon}(t, x)-\widetilde{V}_{\tau}(t, x)\right|=0
$$

as $L_{\tau, \epsilon}\left(t_{0}, x_{0}, \widetilde{u}_{0}, \omega\right) \rightarrow L_{\tau}\left(t_{0}, x_{0}, \widetilde{u}_{0}\right)$ for each $\left(t_{0}, x_{0}, \widetilde{u}_{0}\right) \in[0, T] \times \mathbb{R}^{d} \times \mathbb{R}^{d}$ almost surely and $L_{\tau}$ is uniformly continuous.

Taking $\epsilon \rightarrow 0$ and using Lemma 2.14, we have then that, on the event $\Omega^{\prime}$,

$$
\limsup _{\epsilon \rightarrow 0}\left|V_{\epsilon}(t, x)-\widetilde{V}(t, x)\right| \leq\left|\widetilde{V}(t, x)-\widetilde{V}_{\tau}(t, x)\right|+\limsup _{\epsilon \rightarrow 0}\left|V_{\epsilon}(t, x)-V_{\tau, \epsilon}(t, x, \omega)\right| .
$$

As $\tau$ is arbitrary, we have the result by Lemmas 2.14, 2.15. Uniform convergence on compact sets follows from equicontinunity of $V_{\epsilon}(t, x)$.

\subsection{Homogenization of the Hamiltonian Jacobi equation}

In this subsection we detail the equivalence of homogenizing our control problem, to homogenizing the associated class of Hamilton Jacobi equation. This thereby proves Theorem 1.11.

If we define the Hamiltonians

$$
\begin{aligned}
\mathcal{H}\left(t, x, \frac{x(s)}{\epsilon}, p, \omega\right) & :=\sup _{v \in \mathbb{R}^{d}}\left\{-f(x, v) \cdot p-L\left(t, x, \frac{x}{\epsilon}, v, \omega\right)\right\}, \\
\widetilde{\mathcal{H}}(t, x, p) & :=\sup _{v \in \mathbb{R}^{d}}\{-f(x, v) \cdot p-\widetilde{L}(t, x, v)\},
\end{aligned}
$$

then it is a well known fact that the value functions $V_{\epsilon}$ and $\widetilde{V}$ are the unique viscosity solutions to the following Hamilton-Jacobi equations: ${ }^{2}$

$$
\begin{aligned}
-\frac{\partial V_{\epsilon}}{\partial t}+\mathcal{H} & \left(t, x, \frac{x(s)}{\epsilon}, D_{x} V_{\epsilon}, \omega\right)=0, \quad V_{\epsilon}(T, x, \omega)=\psi(x) \\
-\frac{\partial \widetilde{V}}{\partial t}+\widetilde{\mathcal{H}}\left(t, x, D_{x} \widetilde{V}\right) & =0, \quad \widetilde{V}(T, x)=\psi(x)
\end{aligned}
$$

Thus, having proved that $V_{\epsilon} \rightarrow \widetilde{V}$ uniform on compact subsets, Theorem 1.11 is immediate.

\section{Proof of technical Lemmas}

\subsection{Approximation by piecewise constant dynamics}

Proof of Lemma 2.1.

Step 1: Without loss of generality take $t=0$. Our first step is to prove that we can approximate the value function using controls that are step functions. Fix a $(t, x) \in[0, T] \times \mathbb{R}^{d}$ and a control $u \in \mathcal{U}$. We know from standard analysis (e.g. see [19], Prop. 22, p. 69.) that for any given $\kappa>0$ we can take $\bar{u}$ to be a step function such that,

$$
|\bar{u}(s)-u(s)| \leq \kappa
$$

\footnotetext{
${ }^{2}$ We refer the reader to [9] page 104 for details. We mention that the continunity of the Lagrangians, state dynamics and effective compactness of the control space are essential ingredients.
} 
except perhaps on a set of measure less then $\kappa$ and $\|\bar{u}\|_{\infty} \leq\|u\|_{\infty}$. Denoting $\bar{x}(\cdot)$ as the trajectory of the control $\bar{u}(\cdot)$ we have that

$$
\begin{aligned}
|J(0, x, u)-J(0, x, \bar{u})| & \leq \int_{0}^{\bar{T}}|\bar{L}(s, x(s), u(s))-\bar{L}(s, \bar{x}(s), \bar{u}(s))| \mathrm{d} s \\
& \leq \int_{0}^{\bar{T}} m_{\bar{L}}^{\|u\|_{\infty}}(|\bar{x}(s)-x(s)|) \mathrm{d} s+\kappa \bar{T}\|L\|_{L i p}^{\|u\|_{\infty}}+\kappa L^{*}\left(\|u\|_{\infty}\right) .
\end{aligned}
$$

We then apply Gronwall's inequality on $|\bar{x}(s)-x(s)|$. Specifically for each $t \in[0, \bar{T}]$,

$$
\begin{aligned}
|\bar{x}(s)-x(s)| & \leq \int_{0}^{\bar{T}}|\bar{f}(x(r), u(r))-\bar{f}(\bar{x}(r), \bar{u}(r))| \mathrm{d} r \\
& \leq\|f\|_{\text {Lip }}^{\|u\|_{\infty}} \int_{0}^{\bar{T}}(|\bar{x}(r)-x(r)|+|\bar{u}(r)-u(r)|) \mathrm{d} r \\
& \leq\|f\|_{\text {Lip }}^{\|u\|_{\infty}} \int_{0}^{\bar{T}}(|\bar{x}(r)-x(r)|) \mathrm{d} r+\kappa(T+1) .
\end{aligned}
$$

Therefore $|\bar{x}(s)-x(s)| \leq \kappa(\bar{T}+1) \exp \left(\|f\|_{L i p}^{\|u\|_{\infty}}(T+1)\right)$ for all $t$ substituting this into equation (3.3), we deduce that

$$
|J(0, x, u)-J(0, x, \bar{u})| \leq \bar{T} m_{L}^{\|u\|_{\infty}}\left(\kappa\left|(\bar{T}+1) \exp \left(\|f\|_{L i p}^{\|u\|_{\infty}}(\bar{T}+1)\right)\right|\right)+\kappa \bar{T}\|L\|_{L i p}^{\|u\|_{\infty}}+\kappa L^{*}\left(\|u\|_{\infty}\right) .
$$

This completes step one.

Step 2: In this step we use Assumption 1.8 to show that step one can be converted into the statement about elements of $\mathcal{S}$. Let $\bar{u}(s)$ be as above with $\bar{u}(s)=\bar{u}_{i}$ on $\left[t_{i}, t_{i+1}\right)$. For $i \leq N$, define the quantity $\widetilde{v}_{i}$ as

$$
\widetilde{v}_{i}=\frac{x\left(t_{i+1}\right)-x\left(t_{i}\right)}{t_{i+1}-t_{i}}=\frac{1}{t_{i+1}-t_{i}} \int_{t_{i}}^{t_{i+1}} \bar{f}(x(s), u(s)) \mathrm{d} s .
$$

Note that this is the average of the velocity produced by the original control, $u$. Without loss of generality we can take the time interval of the step function $\bar{u}(s)$ so small so that $\sup _{i \leq N}\left|t_{i+1}-t_{i}\right| f^{*}\left(\|u\|_{\infty}\right) \leq \eta\left(\|u\|_{\infty}\right)$. Then using Assumption 1.8 and 1.9 there exists a control $\widetilde{u}$ such that

$$
\bar{f}(\widetilde{x}(s), \widetilde{u}(s))=\widetilde{v_{i}}
$$

for $s \in\left[t_{i}, t_{i+1}\right)$, where $\widetilde{x}(s)$ is piecewise linear trajectory from $x\left(t_{i}\right)$ to $x\left(t_{i+1}\right)$. We then extend this control to all $[0, \bar{T}]$. Observe in particular that $x\left(t_{i}\right)=\widetilde{x}\left(t_{i}\right)$ and thus $\widetilde{x}$ will also satisfy the same terminal conditions (and have identical terminal cost). Then we have that

$$
\begin{aligned}
|x(t)-\widetilde{x}(t)| & \leq|\bar{x}(t)-x(t)|+|\widetilde{x}(t)-\bar{x}(t)| \\
& \leq\|f\|_{\text {Lip }}^{\|u\|_{\infty}} \int_{0}^{t}(|\bar{x}(s)-\widetilde{x}(s)|+|\bar{u}(s)-\widetilde{u}(s)|) \mathrm{d} s+\kappa(\bar{T}+1) \exp \left(\|f\|_{L i p}^{\|u\|_{\infty}}(\bar{T}+1)\right) .
\end{aligned}
$$

By Gronwall's inequality

$$
|\bar{x}(t)-\widetilde{x}(t)| \leq\left(\int_{0}^{t}|\bar{u}(s)-\widetilde{u}(s)| \mathrm{d} s+\kappa(\bar{T}+1)\right) \exp \left(2\|f\|_{L i p}^{\|u\|_{\infty}}(\bar{T}+1)\right)
$$


Thus we want to acquire a bound on $|\bar{u}(s)-\widetilde{u}(s)|$. But, because $\bar{u}$ is a step function, then we have that, except perhaps on a set of measure less then $\kappa$ (using $|\bar{u}(s)-u(s)| \leq \kappa)$,

$$
\left|\bar{f}(x(s), u(s))-f\left(\bar{x}\left(t_{i}\right), u_{i}\right)\right| \leq\|f\|_{L i p}^{\|u\|_{\infty}}\left|t_{i+1}-t_{i}\right|\left(f^{*}\left(\|u\|_{\infty}\right)+\kappa\right) .
$$

This implies that, if we integrate over $\left[t_{i}, t_{i+1}\right)$ we get

$$
\left|\bar{f}\left(\bar{x}\left(t_{i}\right), u_{i}\right)-\widetilde{v}_{i}\right| \leq\|f\|_{L i p}^{\|u\|_{\infty}}\left(\left|t_{i+1}-t_{i}\right| f^{*}\left(\|u\|_{\infty}\right)+\kappa\right)+\kappa .
$$

Thus,

$$
\begin{aligned}
\left|\bar{f}\left(x(s), u_{i}\right)-\widetilde{v}_{i}\right| & \leq\|f\|_{\text {Lip }}^{\|u\|_{\infty}}\left|t_{i+1}-t_{i}\right| f^{*}\left(\|u\|_{\infty}\right)+\left|f\left(\bar{x}\left(t_{i}\right), u_{i}\right)-\widetilde{v}_{i}\right| \\
& \leq 2\|f\|_{\text {Lip }}^{\|u\|_{\infty}}\left|t_{i+1}-t_{i}\right| f^{*}\left(\|u\|_{\infty}\right)+\kappa\left(\|f\|_{\text {Lip }}^{\|u\|_{\infty}}+1\right) .
\end{aligned}
$$

Then we can utilize Assumption 1.8 to deduce that

$$
|\bar{u}(s)-\widetilde{u}(s)| \leq\|H\|_{L i p}^{\|u\|_{\infty}}\left[2\|f\|_{L i p}^{\|u\|_{\infty}}\left|t_{i+1}-t_{i}\right| f^{*}\left(\|u\|_{\infty}\right)+\kappa\left(\|f\|^{\|u\|_{\infty}}+1\right)\right] .
$$

Choosing these functions such that $\sup _{i}\left|t_{i+1}-t_{i}\right|$ and $\kappa$ tends to 0 and placing them in equation (3.12) and then the equivalent of equation (3.3) with $\bar{x}$ replaced by $\widetilde{x}$ gives the result.

An important point is that this lemma applies to the value function $V_{\epsilon}$ as it can be interpreted as the value function associated with the Lagrangian

$$
\bar{L}_{\epsilon}(t, x, u):=L\left(t, x, \frac{x}{\epsilon}, u, \omega\right)
$$

which is uniformly continuous for each fixed $\epsilon$. Although its modulus of continuity deteriorates as $\epsilon \rightarrow 0$, this is not an issue. The effect of the deteriorating modulus of continuity is that the step functions will gain in complexity. However, in the case where we use then for effective boundedness this does not become a problem. In the other case when we use it to prove Lemma 2.15, the Lagrangian $\widetilde{L}$ is uniformly continuous.

In particular, what will we do in the proof of Lemmas 2.2 and 2.3 is, for any control $u_{n} \in \mathcal{S}$, we will take the time intervals $\left[t_{i}, t_{i+1}\right)$, to be so small that $\left|u_{n}(s)-u_{n}(t)\right| \leq \lambda$ for all $t, s \in\left[t_{i}, t_{i+1}\right)$ for each $i$, where the term $\lambda$ is as in Assumption 1.7. Our ability to do this depends on the function $H$ and consequently will depend also on $\|u\|_{\infty}$.

\subsection{Approximation by bounded control}

Lemma 3.1. Let $\left[t_{0}, t_{0}+h\right] \subset[t, T]$ and $u \in \mathcal{S}$ be such that

$$
\begin{aligned}
& f(x(s), u(s))=\sum_{i=0}^{N} v_{i} 1_{\left[t_{i}, t_{i+1}\right)}(s), \\
& \max _{0 \leq j \leq N} \sup _{s, r \in\left[t_{j}, t_{j+1}\right]}|u(s)-u(r)| \leq \lambda, \\
& \int_{t_{0}}^{t_{0}+h} L\left(s, x(s), \frac{x(s)}{\epsilon}, u(s)\right) \mathrm{d} s \leq W h
\end{aligned}
$$


for some $W>0$, where $\lambda$ is as in Assumption 1.7. Then for sufficiently large $R>0$ depending only on $W$, there exists a control $u_{R} \in \mathcal{U}^{R}$ such that

$$
\int_{t_{0}}^{t_{0}+h} L\left(s, x_{R}(s), \frac{x_{R}(s)}{\epsilon}, u_{R}(s)\right) \mathrm{d} s \leq \int_{t_{0}}^{t_{0}+h} L\left(s, x(s), \frac{x(s)}{\epsilon}, u(s)\right) \mathrm{d} s
$$

and $x\left(t_{0}+h\right)=x_{R}\left(t_{0}+h\right)$.

Proof. We assume $t_{0}=0$ without loss of generality. The assumption $L_{*}(0)>-\infty$ implies that the Lagrangian $L$ is bounded from below. Since we are dealing with a finite time horizon problem, we assume $L \geq 0$ by adding a constant if necessary. The random environment and fast variable are superfluous. For some given $\omega \in \Omega$, we will throughout this proof write the short hand

$$
L_{\epsilon}(t, x, u)=L\left(t, x, \frac{x}{\epsilon}, u, \omega\right)
$$

Let $\left[t_{j}, t_{j+1}\right)$ be the last time period such that $\max _{s \in\left[t_{j}, t_{j+1}\right]}|u(s)| \geq R$. If there is no such time period for $R$ sufficiently large, there is nothing to prove. Define $\hat{t}_{j}:=\operatorname{argmin}_{s \in\left[t_{j}, t_{j+1}\right]}|u(s)|$. By (3.20), we have $\sup _{s \in\left[t_{j}, t_{j+1}\right]}|u(s)| \leq\left|u\left(\hat{t}_{j}\right)\right|+\lambda$ and hence

$$
\left|v_{j}\right|=\left|f\left(x\left(t_{j}\right), u\left(t_{j}\right)\right)\right| \leq \frac{L_{*}\left(\left|u\left(\hat{t}_{j}\right)\right|\right)}{\gamma\left(\left|u\left(\hat{t}_{j}\right)\right|\right)} \leq \frac{L_{*}\left(\left|u\left(\hat{t}_{j}\right)\right|\right)}{\gamma(R-\lambda)},
$$

where $\gamma$ is defined in Assumption 1.7. We divide the proof into 7 steps.

Step 1: Fix an $N>0$ and set

$$
\zeta_{N}(r):=|\{s \leq r:|u(s)| \leq N\}| .
$$

If we take $N$ sufficiently large depending on $W$, we have $\zeta_{N}(h) \geq h / 2$.

Proof of Step 1. Observe that

$$
\int_{\{s \leq r:|u(s)|>N\}} L_{\epsilon}(s, x(s), u(s), \omega) \mathrm{d} s \geq L_{*}(N)\left(h-\zeta_{N}(h)\right) .
$$

On the other hand, since $L$ is assumed to be non-negative,

$$
\int_{\{s \leq r:|u(s)| \leq N\}} L_{\epsilon}(s, x(s), u(s)) \mathrm{d} s \leq W h .
$$

Combining (3.25) and (3.26), we arrive at

$$
h-\zeta_{N}(h) \leq \frac{W h}{L_{*}(N)}
$$

and, since $\lim _{N \rightarrow \infty} L_{*}(N)=\infty$, we are done.

Step 2: We can make

$$
\frac{1}{h} \int_{t_{j}}^{t_{j+1}} f(x(s), u(s)) \mathrm{d} s=\left(t_{j+1}-t_{j}\right) \frac{v_{j}}{h}
$$


as small as we wish by choosing $R$ large depending only on $W$.

Proof of Step 2. By the same way as in the proof of Step 1, we obtain

$$
\left(t_{j+1}-t_{j}\right) L_{*}\left(\left|u\left(\hat{t}_{j}\right)\right|\right) \leq \int_{t_{j}}^{t_{j+1}} L_{*}(|u(s)|) \mathrm{d} s \leq W h .
$$

Using this bound in (3.23), we obtain

$$
\left(t_{j+1}-t_{j}\right)\left|v_{j}\right| \leq \frac{W h}{\gamma(R-\lambda)}
$$

and thanks to Assumption 1.7, we are done.

Step 3: In this step, we construct a time-change function. For $N<R-\lambda$ and $\beta>0$, define

$$
\rho(s)=\int_{0}^{s}\left(1_{r \notin\left[t_{j}, t_{j+1}\right)}-\beta 1_{u(r)<N}+\frac{\left|v_{j}\right|}{\delta} 1_{r \in\left[t_{j}, t_{j+1}\right)}\right) \mathrm{d} r .
$$

If $s \geq t_{j+1}$ this is equal to

$$
s-\beta \zeta_{N}(s)+\left(t_{j+1}-t_{j}\right) \frac{\left|v_{j}\right|}{\delta} .
$$

We choose

$$
\beta=\left(t_{j+1}-t_{j}\right) \frac{\left|v_{j}\right|}{\delta \zeta_{N}(h)}
$$

so that $\rho(h)=h$. By Steps 1 and 2 , we can make $\beta$ as small as we wish by choosing $R>0$ large depending only on $W$ and we always assume $\beta<1$. Our time-change $\sigma$ is defined as the inverse function of $\rho$. Note that $\rho(h)=h$ implies $\sigma(h)=h$. Noting also that $\min _{r \in\left[t_{j}, t_{j+1}\right)}|u(r)| \geq R-\lambda \geq N$, we have

$$
\begin{aligned}
\frac{\mathrm{d} \sigma}{\mathrm{d} s}(s) & =\left(\frac{\mathrm{d} \rho}{\mathrm{d} s}(\sigma(s))\right)^{-1} \\
& =\frac{1_{\sigma(s) \notin\left[t_{j}, t_{j+1}\right)}}{1-\beta 1_{u(\sigma(s))<N}}+\frac{\delta}{\left|v_{j}\right|} 1_{\sigma(s) \in\left[t_{j}, t_{j+1}\right)} .
\end{aligned}
$$

The time-changed trajectory $x \circ \sigma$ has the velocity

$$
\begin{aligned}
\frac{\mathrm{d} x(\sigma(s))}{\mathrm{d} s} & =\frac{\mathrm{d} x}{\mathrm{~d} s}(\sigma(s)) \frac{\mathrm{d} \sigma}{\mathrm{d} s}(s) \\
& =f(x(\sigma(s)), u(\sigma(s))) \frac{\mathrm{d} \sigma}{\mathrm{d} s}(s),
\end{aligned}
$$

which means that we speed up by the factor $(1-\beta)^{-1}$ when $|u(\sigma(s))|<N$ and change the speed to $\delta$ when $\sigma(s) \in\left[t_{j}, t_{j+1}\right)$.

Step 4: When $R>0$ is sufficiently large depending on $W$, there exists a control $\bar{u} \in \mathcal{U}$ which satisfies the following: 
1. The associated trajectory is $x \circ \sigma$, that is,

$$
f(x(\sigma(s)), \bar{u}(s))=f(x(\sigma(s)), u(\sigma(s))) \frac{\mathrm{d} \sigma}{\mathrm{d} s}(s),
$$

2. when $\sigma(s) \in\left[t_{j}, t_{j+1}\right),|\bar{u}(s)| \leq M$,

3. when $|u(\sigma(s))|<N$,

$$
|u(\sigma(s))-\bar{u}(s)| \leq \frac{\beta}{1-\beta}\|H\|_{L i p}^{N} f^{*}(N)
$$

and in particular $|\bar{u}(s)| \leq R$.

Remark 3.2. By the second condition, the control is made small on the last interval where it exceeded $R$. The third condition ensures that we have not created a new point where the control exceeds $R$, i.e., $|\bar{u}|>R$ only on $\bigcup_{i<j} \sigma^{-1}\left(\left[t_{i}, t_{i+1}\right)\right)$.

Proof of Step 4. First, when $\sigma(s) \notin\left[t_{j}, t_{j+1}\right)$ and $|u(\sigma(s))| \geq N$, we have $\frac{\mathrm{d} \sigma}{\mathrm{d} s}(s)=1$ and we can take $\bar{u}(s)=$ $u(\sigma(s))$.

Second, when $\sigma(s) \in\left[t_{j}, t_{j+1}\right)$, since the right hand side is on $\delta \mathbb{S}^{d}$, we can use (1.43) to find $\bar{u}(s) \in U^{M}$ satisfying (3.36). Since $M$ is the constant fixed in (1.43), we have $|\bar{u}(s)| \leq R$ by choosing $R>M$.

Finally, when $\sigma(s) \notin\left[t_{j}, t_{j+1}\right)$ and $|u(\sigma(s))| \leq N$, the right hand side of (3.36) is a small perturbation of $f(x(\sigma(s)), u(\sigma(s)))$. Hence we can use Assumption 6 to find $\bar{u}(s) \in U$ satisfying (3.36): specifically, the $R$ in Assumption 1.8 is set to be $N$ and then we take our $R$ so large (i.e., $\beta$ small) that

$$
\frac{1}{1-\beta} f(x(\sigma(s)), u(\sigma(s))) \in B_{\eta(N)}(f(x(\sigma(s)), u(\sigma(s)))) .
$$

Then we can define the desired control as

$$
\bar{u}(s)=H\left(x(\sigma(s)), \frac{1}{1-\beta} f(x(\sigma(s)), u(\sigma(s)))\right) .
$$

Recalling the identity $H(x(\sigma(s)), f(x(\sigma(s)), u(\sigma(s))))=u(\sigma(s))$ and the Lipschitz continuity of $H$ in Assumption 1.8 , we have

$$
\begin{aligned}
|u(\sigma(s))-\bar{u}(s)| & \leq\|H\|_{L i p}^{N}\left(\frac{1}{1-\beta}-1\right)|f(x(\sigma(s)), u(\sigma(s)))| \\
& \leq\|H\|_{L i p}^{N} \frac{\beta}{1-\beta} f^{*}(N) .
\end{aligned}
$$

Taking $R$ large makes $\beta$ small and we can conclude $|\bar{u}(s)| \leq R$.

Step 5: The following hold:

$$
\begin{aligned}
& \left|\left\{s: \sigma(s) \in\left[t_{j}, t_{j+1}\right)\right\}\right|=\left(t_{j+1}-t_{j}\right) \frac{\left|v_{j}\right|}{\delta}, \\
& \sup _{s \in[0, h]}|\sigma(s)-s| \leq \frac{\beta}{1-\beta} h+\left(t_{j+1}-t_{j}\right) \frac{\left|v_{j}\right|}{\delta} .
\end{aligned}
$$


Proof of Step 5. The first claim is a consequence of the fact that $x \circ \sigma$ travels the distance $\left(t_{j+1}-t_{j}\right)\left|v_{j}\right|$ in speed $\delta$. For the proof of the second claim, by (3.34), we know that $\frac{\mathrm{d} \sigma}{\mathrm{d} s}(s) \neq 1$ only if $u(\sigma(s))<N$ or $\sigma(s) \in\left[t_{j}, t_{j+1}\right)$. Therefore,

$$
\begin{aligned}
|\sigma(s)-s| & \leq \int_{0}^{s}\left|\frac{\mathrm{d} \sigma}{\mathrm{d} s}(s)-1\right| \mathrm{d} s \\
& \leq\left(\frac{1}{1-\beta}-1\right) \zeta_{N}(h)+\left|\frac{\delta}{\left|v_{j}\right|}-1\right|\left|\left\{s: \sigma(s) \in\left[t_{j}, t_{j+1}\right)\right\}\right| .
\end{aligned}
$$

Substituting the first claim and $\zeta_{N}(h) \leq h$, we obtain (3.42).

Step 6: When $R>0$ is sufficiently large depending on $W$,

$$
\int_{0}^{h} L(s, x(s), u(s)) \mathrm{d} s \geq \int_{0}^{h} L_{\epsilon}(s, \bar{x}(s), \bar{u}(s)) \mathrm{d} s .
$$

Proof of Step 6 . We assume $\frac{\beta}{1-\beta}<2 \beta$ by choosing $R>0$ large enough. The left hand side is bounded from below by

$$
\left(t_{j+1}-t_{j}\right) L_{*}\left(u\left(\hat{t}_{j}\right)\right)+\int_{0}^{t_{j}} L_{\epsilon}(s, x(s), u(s)) \mathrm{d} s+\int_{t_{j+1}}^{h} L_{\epsilon}(s, x(s), u(s)) \mathrm{d} s .
$$

Making the substitution $s=\sigma(r)$, recalling $x(\sigma(r))=\bar{x}(r)$ and noting that $\sigma^{\prime}(r) \geq 1$ on the above two domains of integration, we can bound the second term from below by

$$
\int_{0}^{\sigma^{-1}\left(t_{j}\right)} L_{\epsilon}(\sigma(r), \bar{x}(r), u(\sigma(r))) \sigma^{\prime}(r) \mathrm{d} r \geq \int_{0}^{\sigma^{-1}\left(t_{j}\right)} L_{\epsilon}(\sigma(r), \bar{x}(r), u(\sigma(r))) \mathrm{d} r
$$

Applying the same argument to the third term in (3.45), we find that the sum of two integrals in (3.45) is bounded from below by

$$
\int_{\left[0, \sigma^{-1}\left(t_{j}\right)\right) \cup\left[\sigma^{-1}\left(t_{j+1}\right), h\right]} L_{\epsilon}(\sigma(r), \bar{x}(r), u(\sigma(r))) \mathrm{d} r .
$$

Using Steps 4 and 5, we can evaluate the error of replacing $\sigma(r)$ and $u(\sigma(r))$ by $r$ and $\bar{u}(r)$ in this integral and obtain

$$
\begin{aligned}
& \int_{\left[0, \sigma^{-1}\left(t_{j}\right)\right) \cup\left[\sigma^{-1}\left(t_{j+1}\right), h\right]} L_{\epsilon}(\sigma(r), \bar{x}(r), u(\sigma(r))) \mathrm{d} r \\
& \quad \geq \int_{\left[0, \sigma^{-1}\left(t_{j}\right)\right) \cup\left[\sigma^{-1}\left(t_{j+1}\right), h\right]} L_{\epsilon}(r, \bar{x}(r), \bar{u}(r)) \mathrm{d} r \\
& \quad-h\|L\|_{L i p}\left(2 \beta h+\left(t_{j+1}-t_{j}\right) \frac{\left|v_{j}\right|}{\delta}\right)-h\|L\|_{L i p}^{N} \times 2 \beta\|H\|_{L i p}^{N} f^{*}(N) \\
& \quad=\int_{\left[0, \sigma^{-1}\left(t_{j}\right)\right) \cup\left[\sigma^{-1}\left(t_{j+1}\right), h\right]} L_{\epsilon}(r, \bar{x}(r), \bar{u}(r)) \mathrm{d} r-c(W)\left(t_{j+1}-t_{j}\right) \frac{\left|v_{j}\right|}{\delta},
\end{aligned}
$$


where in the last line we have used the definition (3.33) of $\beta$ and Step 1. (Recall that $N$ depends only on $W$.) On the remaining interval $\left[\sigma^{-1}\left(t_{j}\right), \sigma^{-1}\left(t_{j+1}\right)\right)$, we have $|\bar{u}| \leq M$ by Step 4 and hence combining with Step 5 ,

$$
\int_{\left[\sigma^{-1}\left(t_{j}\right), \sigma^{-1}\left(t_{j+1}\right)\right)} L_{\epsilon}(r, \bar{x}(r), \bar{u}(r)) \mathrm{d} r \leq L^{*}(M)\left(t_{j+1}-t_{j}\right) \frac{\left|v_{j}\right|}{\delta} .
$$

Substituting all the above estimates to (3.45), we find that

$$
\begin{aligned}
\int_{0}^{h} L_{\epsilon}(s, x(s), u(s)) \mathrm{d} s \geq & \int_{0}^{h} L_{\epsilon}(r, \bar{x}(r), \bar{u}(r)) \mathrm{d} r \\
& +\left(t_{j+1}-t_{j}\right)\left(L_{*}\left(u\left(\hat{t}_{j}\right)\right)-\left(c(W)+L^{*}(M)\right) \frac{\left|v_{j}\right|}{\delta}\right) .
\end{aligned}
$$

By (3.23) and Assumption 1.7, the second term on the right hand side is positive for sufficiently large $R>0$ depending only on $W$.

Step 7: We can apply the above procedure to $\bar{u}$ again and continue recursively. As we noticed in Remark 3.2, this procedure always decreases the number of intervals where the control exceeds $R$. Therefore, after finitely many steps, we end up with a new control $u_{R} \in \mathcal{U}^{R}$ that gives a smaller cost than the original one.

Remark 3.3. As we proved in Section 2.5, the same estimates on the homogenized control problem as well as the discrete problems are applicable. Hence we can easily extend Lemma 3.1 to control problems with Lagrangians $\widetilde{L}, L_{\tau, \epsilon}$.

Using Lemma 3.1 we can now readily prove Lemmas 2.2, 2.3 and 2.13.

Proof of Lemma 2.3. For the object $\widehat{L}_{\tau, \epsilon}\left(t_{0}, x_{0}, \widetilde{u}_{0}, \omega\right)$, if we consider the control $u(s)$ such that $f(x(s), u(s))=$ $f\left(x_{0}, \widetilde{u}\right)$, then we conclude that

$$
\widehat{L}_{\tau, \epsilon}\left(t_{0}, x_{0}, \widetilde{u}_{0}, \omega\right) \leq h L^{*}\left(\left|\widetilde{u}_{0}\right|+\eta\left(\left|\widetilde{u}_{0}\right|\right)\right)
$$

by taking $W=L^{*}\left(\left|\widetilde{u}_{0}\right|+\eta\left(\left|\widetilde{u}_{0}\right|\right)\right)$. If $u_{n}$ is a sequence of controls such that

$$
\lim _{n \rightarrow \infty} \int_{t_{0}}^{t_{0}+h} L\left(t_{0}, x_{0}, \frac{x(s)}{\epsilon}, u_{n}(s), \omega\right) \mathrm{d} s=\widehat{L}_{\tau, \epsilon}\left(t_{0}, x_{0}, \widetilde{u}_{0}, \omega\right) \leq h L^{*}\left(|\widetilde{u}|+\eta\left(\left|\widetilde{u}_{0}\right|\right)\right)
$$

which we can without loss of generality take $u_{n} \in \mathcal{S}$ by Lemma 2.1, then by Lemma 3.1 we find a sequence of controls $\bar{u}_{n}(s)$ such that $\bar{u}_{n}(s) \in \mathcal{U}^{K_{1}}$ for some $K_{1}$ depending only on $L^{*}\left(\left|\widetilde{u}_{0}\right|+\eta\left(\left|\widetilde{u}_{0}\right|\right)\right)$ and

$$
\int_{t_{0}}^{t_{0}+h} L\left(t_{0}, x_{0}, \frac{x(s)}{\epsilon}, \bar{u}_{n}(s), \omega\right) \mathrm{d} s \leq \int_{t_{0}}^{t_{0}+h} L\left(t_{0}, x_{0}, \frac{x(s)}{\epsilon}, u_{n}(s), \omega\right) \mathrm{d} s
$$

and therefore we conclude

$$
\widehat{L}_{\tau, \epsilon}\left(t_{0}, x_{0}, \widetilde{u}_{0}, \omega\right)=\widehat{L}_{\tau, \epsilon}^{K 1}\left(t_{0}, x_{0}, \widetilde{u}_{0}, \omega\right)
$$

It is trivial to repeat the proof of Lemma 3.1 for the frozen dynamics $f\left(x_{0}, \widetilde{u}_{0}\right)$. Then, taking $W=L^{*}\left(\left|\widetilde{u}_{0}\right|\right)$ we can show for some $K_{2}$

$$
L_{\tau, \epsilon}\left(t_{0}, x_{0}, \widetilde{u}_{0}, \omega\right)=L_{\tau, \epsilon}^{K_{2}}\left(t_{0}, x_{0}, \widetilde{u}_{0}, \omega\right)
$$


Taking $K=K_{1} \wedge K_{2}$ and observing that this $K$ depends only on $\widetilde{u}_{0}$ completes the proof.

Proof of Lemma 2.2 and 2.13. Take a sequence of controls $u_{n} \in \mathcal{S}$ such that

$$
\lim _{n \rightarrow \infty} J_{\epsilon}\left(t, x, u_{n}, \omega\right)=V_{\epsilon}(t, x, \omega)
$$

Clearly there exists an $M>0$ such that $J_{\epsilon}\left(t, x, u_{n}, \omega\right) \leq M$ for all $n$. Therefore we can conclude that there exists a $K>0$ and sequence of controls $\bar{u}_{n} \in \mathcal{U}^{K}$ such that

$$
J_{\epsilon}\left(t, x, \bar{u}_{n}, \omega\right) \leq J_{\epsilon}\left(t, x, u_{n}, \omega\right)
$$

in part owing to the fact that $x_{n}(T)=\bar{x}_{n}(T)$ and hence $\psi\left(x_{n}(T)\right)=\psi\left(\bar{x}_{n}(T)\right)$. Thus

$$
V^{K}(t, x, \omega) \leq \lim _{n \rightarrow \infty} J_{\epsilon}\left(t, x, \bar{u}_{n}, \omega\right)=V(t, x, \omega)
$$

The reverse inequality is trivial and hence this completes the proof of Lemma 2.2. Adopting the same argument for $\widetilde{J}$ and $J_{\tau, \epsilon}$ we can immediately obtain Lemma 2.13

Acknowledgements. This research was supported by a Monbukagakusho scholarship administered by the Japanese Ministry of Education, Culture, Sports, Science and Technology. I would also like to thank Ryoki Fukushima for his helpful insight and guidance.

\section{REFERENCES}

[1] S. Armstrong, P. Cardaliaguet and P. Souganidis, Error estimates and convergence rates for the stochastic homogenization of Hamilton-Jacobi equations. J. Amer. Math. Soc. 27 (2014) 479-540.

[2] J. Ball and V.J. Mizel, One-dimensional Variational Problems whose Minimizers do not Satisfy the Euler-Lagrange Equation. Arch. Ratl. Mech. Anal. 90 (1985) 325-388.

[3] A. Davini and A. Siconolfi, Metric techniques for convex stationary ergodic Hamiltonians. Calc. Variat. Partial Differ. Equ. 40 (2011) 391-421.

[4] A. Davini and A. Siconolfi, Weak KAM theory topics in the stationary ergodic setting. Calc. Variat. Partial Differ. Equ. 44 (2012) 319-350.

[5] N. Dirr, F. Dragoni, P. Mannucci and C. Marchi, Stochastic homogenization for functionals with anisotropic rescaling and noncoercive Hamilton-Jacobi equations. SIAM J. Math. Anal. 50 (2018) 5198-5242.

[6] L.C. Evans, The perturbed test function method for viscosity solutions of nonlinear PDE. Proc. Royal Soc. Edinburgh: Sect. A Math. 111 (1989) 359-375.

[7] W.M. Feldman and P.E. Souganidis, Homogenization and non-homogenization of certain non-convex Hamilton-Jacobi equations. J. Math. Pures Appl. 108 (2017) 751-782.

[8] A. Filippov, On certain questions in the theory of optimal control. J. Soc. Ind. Appl. Math. Ser. A Control 1 (1962) 76-84.

[9] W. Fleming and H. Soner, Controlled Markov Processes and Viscosity Solutions, Stochastic Modelling and Applied Probability, Springer New York (2006).

[10] H. Ishii, Almost periodic homogenization of Hamilton-Jacobi equations. Int. Conf. Differ. Equ. 1 (2000).

[11] W. Jing, P.E. Souganidis and H.V. Tran, Stochastic homogenization of viscous superquadratic Hamilton-Jacobi equations in dynamic random environment. Res. Math. Sci. 4 (2017) 6.

[12] E. Kosygina, F. Rezakhanlou and S.R.S. Varadhan, Stochastic homogenization of Hamilton-Jacobi-Bellman equations. Commun. Pure Appl. Math. 59 (2006) 1489-1521.

[13] U. Krengel and A. Brunel, Ergodic Theorems. De Gruyter Studies in Mathematics, De Gruyter (1985).

[14] P.-L. Lions, G. Papanicolaou and S.R. Varadhan, Homogenization of Hamilton-Jacobi equations. unpublished (1986).

[15] P.-L. Lions and P. Souganidis, Correctors for the homogenization of Hamilton-Jacobi equations in the stationary ergodic setting. Commun. Pure Appl. Math. 56 (2003) 1501-1524.

[16] P.-L. Lions and P.E. Souganidis, Homogenization of "viscous" Hamilton-Jacobi equations in stationary ergodic media. Commun. Partial Differ. Equ. 30 (2005) 335-375.

[17] F. Rezakhanlou and J.E. Tarver, Homogenization for stochastic Hamilton-Jacobi equations. Arch. Ratl. Mech. Anal. 151 (2000) 277-309.

[18] E. Roxin, The existence of optimal controls. Michigan Math. J. 9 (1962) 109-119.

[19] H. Royden, Real Analysis. Mathematics and statistics, Macmillan (1988). 
[20] P.E. Souganidis, Stochastic homogenization of Hamilton-Jacobi equations and some applications. Asymptotic Anal. 20 (1999) 1-11.

[21] A. Stoddart, Existence of optimal controls. Pacific J. Math. 20 (1967) 167-177.

[22] B. Ziliotto, Stochastic Homogenization of Nonconvex Hamilton-Jacobi Equations: A Counterexample. Commun. Pure Appl. Math. (2015) arXiv:1512.06375. 